\title{
Comprehensive wind tunnel experiments of lofting and downwind transport of non-combusting rod-like model firebrands during firebrand shower scenarios
}

\author{
Ali Tohidi ${ }^{\mathrm{a}, *}$, Nigel Berkeley Kaye ${ }^{\mathrm{b}}$ \\ ${ }^{a}$ University of Maryland, College Park, MD, USA, 20740 \\ ${ }^{b}$ Clemson University, Clemson, SC, USA, 29634
}

\begin{abstract}
To date, due to difficulties in making measurements during wildfires, much of what is known about firebrand showers and the subsequent fire spotting comes from mathematical modeling of the lofting and downwind transport of firebrands. However, these models lack experimental validation. Hence, the coupled lofting and downwind transport of non-combusting rod-like firebrands is experimentally modeled by releasing them through the velocity field of a large scale boundary layer wind tunnel. Complete trajectories of model firebrands are resolved using image processing algorithms. The results show a strong positive correlation between the maximum rise height $\left(z_{\max }\right)$ and the landing location $\left(x_{l}\right)$ of model firebrands. In addition, it is shown that, given the velocity field, the empirical probability density functions (PDF) of $x_{l} / z_{\max }$ are similar regardless of the firebrands' aspect ratio. This implies that the lofting and downwind transport processes cannot be decoupled in transport models. Analysis of the data reveals that, the larger the aspect ratio of firebrands, the more sensitive their landing locations are to the variability in the velocity field through which they are released. The data set presented herein serves as the most comprehensive experimental evidence for not only firebrand transport studies but also for validating mathematical models for the flight of rod-like debris/brands within
\end{abstract}

\footnotetext{
${ }^{*}$ Corresponding author

Email addresses: atohidi@umd.edu (Ali Tohidi), nbkaye@clemson.edu (Nigel Berkeley Kaye)
}

Preprint submitted to Journal of ${ }^{A} T_{E} X$ Templates

February 7, 2017

(C) 2017. This manuscript version is made available under the Elsevier user license http://www.elsevier.com/open-access/userlicense/1.0/ 
the velocity field of other extreme events such as hurricanes.

Keywords: Wildfires, Firebrand, Wind Tunnel Experiments, Particle

Tracking, Image Processing, Firebrand Aerodynamics, Firebrand Shower, Fire Spotting

1

\section{Introduction}

Firebrand showers, that may lead to spot fire ignition, are not only a perilous and inevitable phenomenon during wildfires but also in urban fires or any large conflagration. There is a growing body of evidence to suggest that firebrand showers are responsible for fire spread [32]. Observations range from the great fire of London (1666) [12, post-earthquake conflagrations of San Francisco (1906) and Tokyo (1923) 25], recent wildfires in Australia and Greece [35, to numerous other cases; see Koo et al. [25], and Tohidi et al. [49]. Spot fires generated by firebrand showers are highly stochastic and, as the intensity and size of fires grow, they become more severe [4. This necessitates understanding of firebrand flight mechanisms leading to firebrand showers, as they pose a major threat to people, properties, and infrastructure, particularly at wildland urban interfaces (WUI) [4].

A typical firebrand shower process is as follows. Once a wildfire or any large conflagration occurs, combustion processes such as pyrolysis and char affect the vegetative structures or woody elements. Due to the subsequent thermomechanical processes firebrands form and break-off from the burning vegetation/structures [11, 49]. Then, firebrands can get lofted up through the fire plume where, if the rise height is sufficient, transition from lofting to downwind transport within the atmospheric boundary layer occurs. For more information on various lofting to downwind transition criteria see Tohidi and Kaye [50]. Critical environmental factors such as topography, direction of the fire line spread, separation due to wind on the lee side of the ridge, and fuel breaks may enhance the air entrainment into the fire plume, increases the updraft and consequently 
intensify the firebrand shower [18, 24]. Ultimately, a spot fire may ignite upon landing on susceptible fuel beds far ahead of the fire front.

The lofting and downwind transport of firebrands through the velocity field generated by the interaction of the fire plume and the atmospheric boundary layer plays a significant role in the fire spotting phenomenon and subsequently wildfire spread. To date many studies have been done on the flight of debris/firebrands through different velocity fields. For instance, in a series of studies Tarifa et al. 45, 44 investigated combustion and transport properties of firebrands along with the influence of their size, shape, density, and moisture content on transport in a small scale wind tunnel. These studies were conducted for a very simplified model of firebrands with no rotational effects. The aerodynamic behavior and trajectories of spherical model firebrands through a naturally convective turbulent swirling plume was discussed by Lee and Hellman [27, 28]. In addition, Tse and Fernandez-Pello [52, Himoto and Tanaka [20, Anthenien et al. [10, Sardoy et al. 41, 39, and Bhutia et al. 13] have modeled fate and transport of different types of firebrands, numerically. Himoto and Tanaka [21] developed and validated an urban fire spread model that, instead of modeling firebrand lofting and transport, uses a probabilistic approach for estimating the firebrand travel distance.

While these studies are of great value in improving our understanding of the processes involved in firebrand transport, a large number of the developed mathematical models are based on simplifying assumptions that raise concerns about their applicability to real fire events. Further, there has been little experimental work [29, 38, 37] done on the rod-like firebrand/debris transport, and majority of the numerical models suffer from lack of thorough experimental validation. The previous works on (rod-like) debris [52, 41, 4, 8, 2, 3, 5, 6, 9, 17, 20, 21, 45, 44, 36] have focused on horizontal wind fields and have not examined the interactions of vertical lofting flow with the horizontal atmospheric boundary layer winds. The main objective of this study is to fill this gap by presenting results of an 
extensive set of large scale wind tunnel experiments for lofting and transport of non-combusting rod-like model firebrands through the scaled-down velocity field of small to medium wildfires.

The experimental results will enable rigorous validation of current and future firebrand flight models. Also, the results have applications beyond firebrand transport, as they provide a broad experimental analysis for transport of rod-like debris with different aspect ratios in a turbulent boundary layer. The remainder of this paper is structured as follows: the experimental setup is discussed in Section 2. In Section 3 the data acquisition technique is presented followed by Section 4 through which the experimental results \& analysis are presented. Concluding remarks are drawn in Section 5 .

\section{Experimental setup}

A set of lofting and transport experiments were conducted to examine the flight characteristics of rod-like firebrands lofted vertically into model wildfire velocity field generated by the interaction of the fire plume and the atmospheric boundary layer.

\subsection{Brief discussion on scaling}

In fluid dynamics, measurements are often performed on models, under controlled conditions, that either share the same characteristics with the actual system or have similitudes with the phenomenon. The concept of similarity permits extension of information in experimental models to the actual phenomena or systems. The essential requirement for complete similarity is that the geometric, kinematic, and dynamic similarity between the model and the phenomenon must be satisfied. However, this is not feasible when one is modeling lofting and transport of firebrands through the velocity field of wildfires. In fact, since very little is known about firebrands generated through a real wildfire, and laboratory studies exhibit a very wide range of sizes and shapes [33, 32, 31, 34, 
the geometric similarity would require an unfeasible amount of experiments. Dynamic similarity is, also, not possible in wind tunnels as pool fires need to be created and then the wind speed scaled to match both the Froude number and plume to wind velocity ratio [51]. For small-scale laboratory fires, even in the largest available wind tunnels, this would lead to very low wind speeds which would lead to a boundary layer that is not in the fully developed turbulent regime.

Therefore, it is not possible to run wind tunnel experiments of firebrand lofting and transport in which geometric and dynamic similarity are satisfied. Instead, a series of wind tunnel tests were run in which model firebrands were lofted into the wind tunnel boundary layer using a vertical air jet. The stream wise firebrand trajectory was then measured. While the use of a vertical nonbuoyant air jet is not directly analogous to a vertical buoyant fire plume the two flows are quite similar. They are both round vertical turbulent shear flows that entrain fluid as they rise due to shear with the surrounding environment. The main difference is that the buoyancy of the plume generates additional vertical momentum as it rises whereas a turbulent jet has a constant vertical momentum flux. This means that a jet will bend horizontally at a lower height compared to a turbulent plume with the same source momentum flux but with an additional buoyancy flux. However, the overall structure of the two flows is similar and the effect of buoyancy on the large scale dynamics can be accounted for in re-scaling [51]. The density of the hot air in the plume will make only a very small difference to the flight trajectory of a firebrand as the buoyancy force acting on the firebrand is negligible. Therefore, the use of a vertical air jet rather than a vertical plume will still provide a valuable model validation data set for firebrand transport models. The main goal of experiments is to develop an experimental data set that can be used to validate mathematical models which can then be applied to full scale fires. More discussion of the use of this data set for firebrand transport model validation will be presented in the final section of this paper. 

rations, i.e. $\eta=L_{z} / L_{x, y}$, as shown in figure 1. Here, $L_{z}$ denotes the major

\subsection{Setup}

Boundary layer wind tunnel experiments of fire spotting were run using non-combusting polyurethane model firebrands with average density of $\varrho=$ $30 \mathrm{~kg} / \mathrm{m}^{3}$, side aspect ratio $\eta_{s}=L_{x} / L_{y}=1$, and different longitudinal aspect

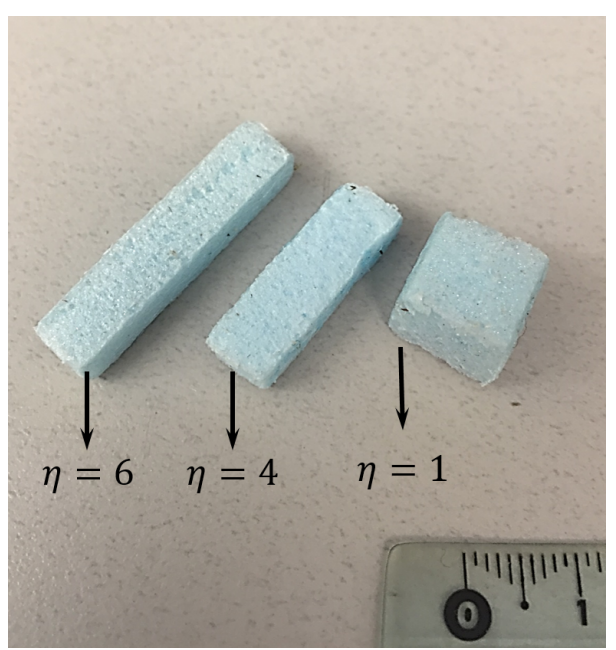

Figure 1: Polyurethane made model firebrands with different aspect ratios; The scale shows $1 \mathrm{~cm}$ in the image space.

side and $L_{x} \& L_{y}$ denote the minor sides. The dimension of all sides in $\eta=1$ model firebrands are equal, i.e. $L_{x}=L_{y}=L_{z}=1 \mathrm{~cm}$, and for the other aspect ratios $L_{x}=L_{y}=0.5 \mathrm{~cm}$. Low density manufactured polyurethane firebrands were used instead of natural wood sections for two reasons. First, the more precise dimensions allowed for greater experimental repeatability, and second, the Polyurethane was light enough to be lofted vertically by the air jet high into the wind tunnel tests section whereas sample wood dowel rods were unable to be lofted.

The tests were conducted in Clemson University's wind tunnel testing facility which is classified as a low-speed boundary layer wind tunnel, as the maximum achievable velocity is less than $100 \mathrm{~m} / \mathrm{s}$ [46]. Hence, flow compressibility effects 

and surface roughness elements were used; see figure 2-left. The spire board

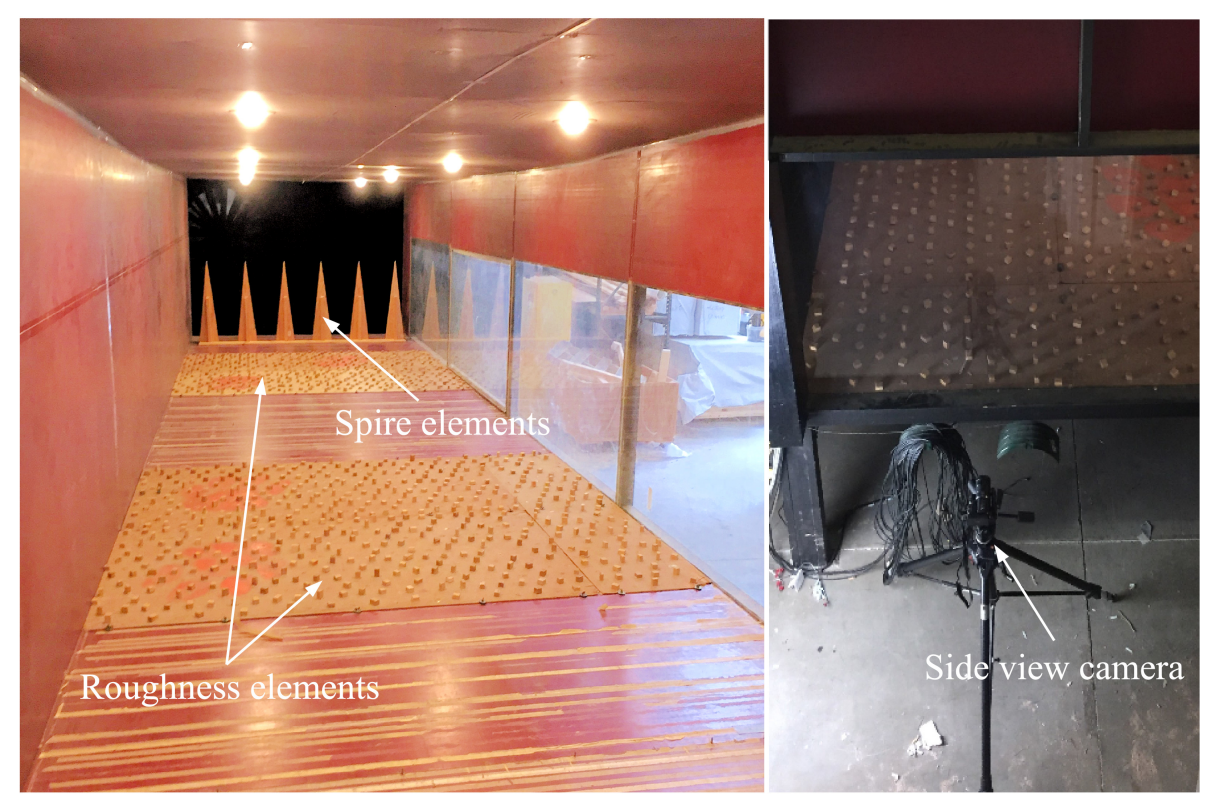

Figure 2: (Left) Inside the wind tunnel chamber where the surface roughness elements and spire elements are installed. Also, on the right, the camera position at the side view of the release point (jet location) is shown.

139

are negligible or very small in the experiments. The test section is comprised of a $3.05 \mathrm{~m}$ wide by $2.03 \mathrm{~m}$ high open boundary layer with $20 \mathrm{~m}$ of fetch. The wind flow is generated by two $1.8 \mathrm{~m}$ diameter fans which are controlled by adjustable frequency inverters. The flow is passed through a honeycomb grid, a set of screens and a contraction to produce low turbulence intensity uniform inflow. In order for the boundary layer to be turbulent, a combination of a spire board consists of 6 triangles with $92.2 \mathrm{~cm}$ height and $25.4 \mathrm{~cm}$ base that were evenly spread across the $3.05 \mathrm{~m}$ width of the wind tunnel immediately downwind of the contraction. The surface roughness elements are made from randomly located rectangular prisms of length $3.2 \mathrm{~cm}$ and thickness of $1.6 \mathrm{~cm}$ that are stuck to boards with glue. The surface roughness boards covered over $11 \mathrm{~m}$ of the upstream section to generate a grass (open flat) terrain exposure [14. For more details on the specifics of the wind tunnel see Liu et al. [30]. 

centerline exit velocity could be adjusted between $0-12 \mathrm{~m} / \mathrm{s}$.

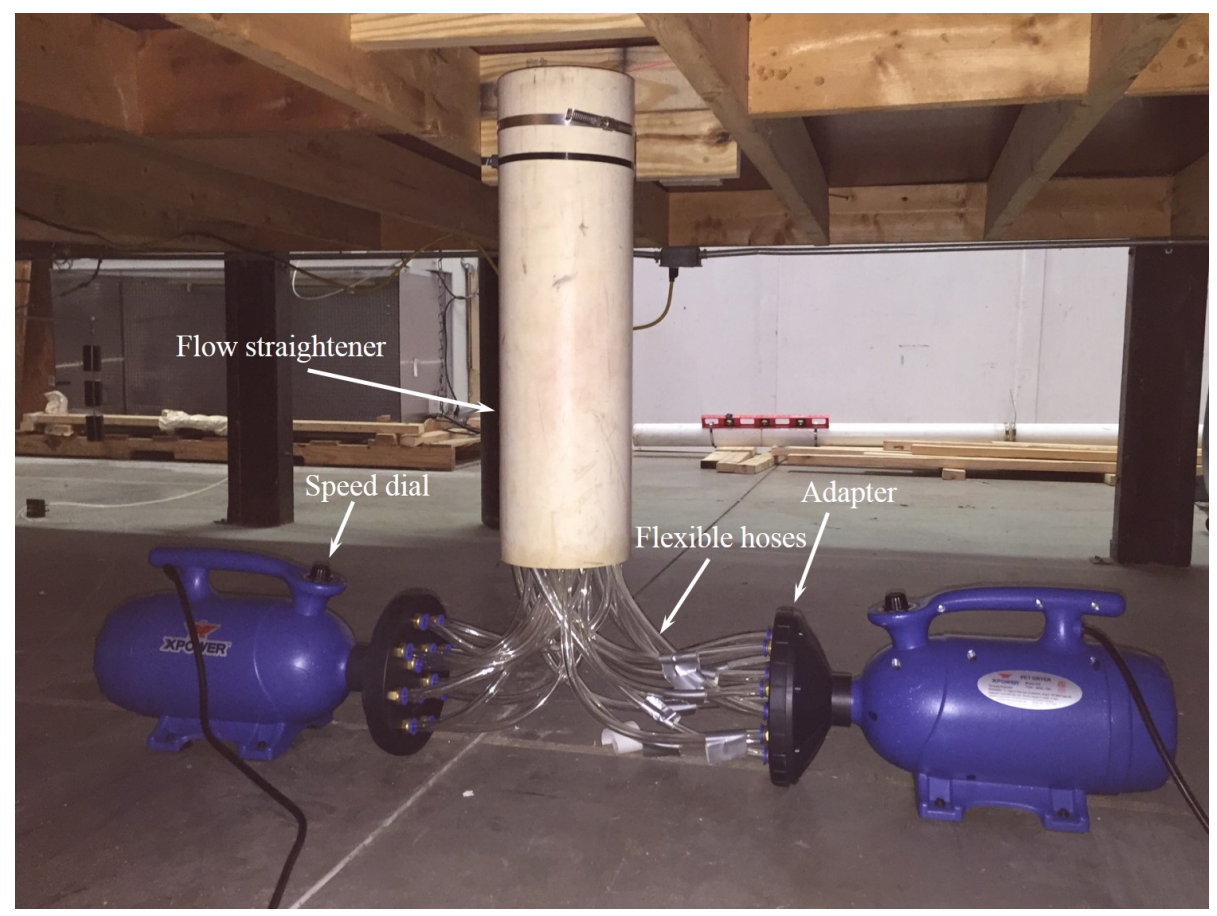

Figure 3: Details of the mounted air jet underneath the wind tunnel floor. Also, shown are blowers, and the flow straightener.

The velocity field of the fire plume was modeled using an air jet installed in the floor of the wind tunnel; see figure 3 . The $15.2 \mathrm{~cm}$ diameter jet was created using two $4 \mathrm{hp}$ blowers that were fed into a $58.8 \mathrm{~cm}$ flow straightener. The jet

blowers and the flow straightener.

Experiments were run for combinations of three boundary layer reference velocities and three jet centerline velocities, in order to model the velocity field induced by the interaction of the fire plume and the boundary layer. The generated velocity fields are measured using an Extech - 407112 heavy duty hot-wire thermo-anemometer which has $\pm 0.1 \mathrm{~m} / \mathrm{s}$ resolution in the range of $0.2-20 \mathrm{~m} / \mathrm{s}$. As for of the boundary layer, the velocity measurements are conducted at the centerline of the wind tunnel test section, exactly $25 \mathrm{~cm}$ upstream of the jet 

available field data for small scale wildfires [13, 51].

Table 1: Specifics of the non-combusting model firebrands' lofting and transport experiments. Here, $U_{0}$ denotes the horizontal component of boundary layer wind tunnel velocity at the reference height of $z_{0}=0.04 \mathrm{~m}$, the jet centerline velocity is shown by $U_{j \mid r=0}, \eta=L_{z} / L x, y$ is the planks (model firebrands) aspect ratio, and D.O.F. stands for the degrees of freedom (nominal number of releases) for each aspect ratio.

\begin{tabular}{c|c|c|c}
\hline \multirow{2}{*}{$1.79(\mathrm{~m} / \mathrm{s})$} & $U_{j \mid r=0}(\mathrm{~m} / \mathrm{s})$ & $\eta$ & D.O.F. \\
\hline \multirow{3}{*}{1.79} & 12 & $1,4,6$ & 200 \\
\cline { 2 - 4 } & 8.05 & $1,4,6$ & 200 \\
\cline { 2 - 4 } & 9 & $1,4,6$ & 200 \\
\hline \multirow{2}{*}{2.23} & 12 & $1,4,6$ & 200 \\
\cline { 2 - 4 } & 8.05 & $1,4,6$ & 200 \\
\cline { 2 - 4 } & 9 & $1,4,6$ & 200 \\
\hline \multirow{2}{*}{2.85} & 12 & $1,4,6$ & 200 \\
\cline { 2 - 4 } & 8.05 & $1,4,6$ & 200 \\
\cline { 2 - 4 } & 9 & $1,4,6$ & 200 \\
\hline
\end{tabular}

centerline when the jet was turned off. Similarly, centerline and radial velocity profiles of the jet were measured with no wind. The cross-sectional velocities were measured at $0.138 \mathrm{~m}$ from the jet nozzle exit, where the jet cross-section is level with the wind tunnel's floor. This small space is utilized for releasing model firebrands through the domain. Specific parameter combinations tested in the experiments are set out in table 1. Also, it should be mentioned that the ratio of jet centerline velocity to the boundary layer reference velocity, i.e. $\left(U_{j \mid r=0} / U_{0}\right)$, is of the order of $4 \pm 1$ for these tests, and corresponds to the sent

In the boundary layer the reference height is taken to be $z_{0}=0.04 \mathrm{~m}$ to fit the power-law profile, namely $U=U_{0}\left(z / z_{0}\right)^{\alpha}$. Sixty second time averaged velocity measurements in a vertical profile showing the boundary layer are presented in figure 4 along with their corresponding power-law fit. Sixty second 


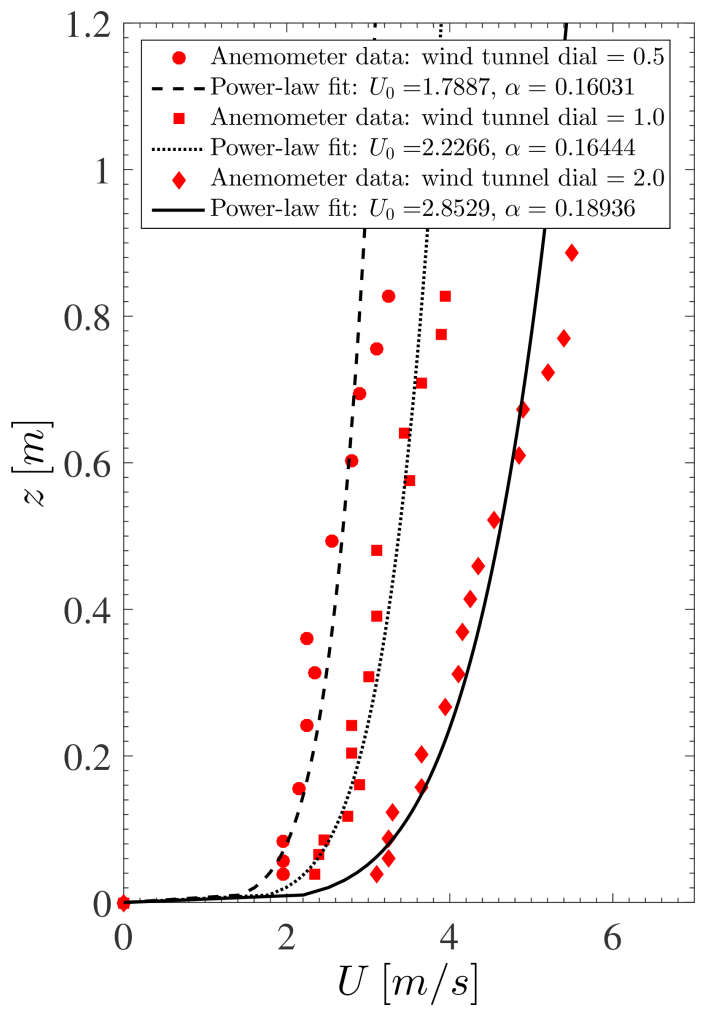

Figure 4: Vertical profiles of the boundary layer horizontal (stream-wise) velocity component measured in the wind tunnel. Also, $U_{0}$ is the horizontal velocity of the corresponding powerlaw fits with exponent $\alpha$ and the reference height $z_{0}=0.04 \mathrm{~m}$.

As can be seen in figure 5-left, the normalized time-averaged vertical (streamwise) velocity profile of the jets are similar and approximately collapse onto a single curve. The time-averaged radial variation in vertical velocity, see figure 5 . right, is less consistent due to different inlet conditions. The measurements are 

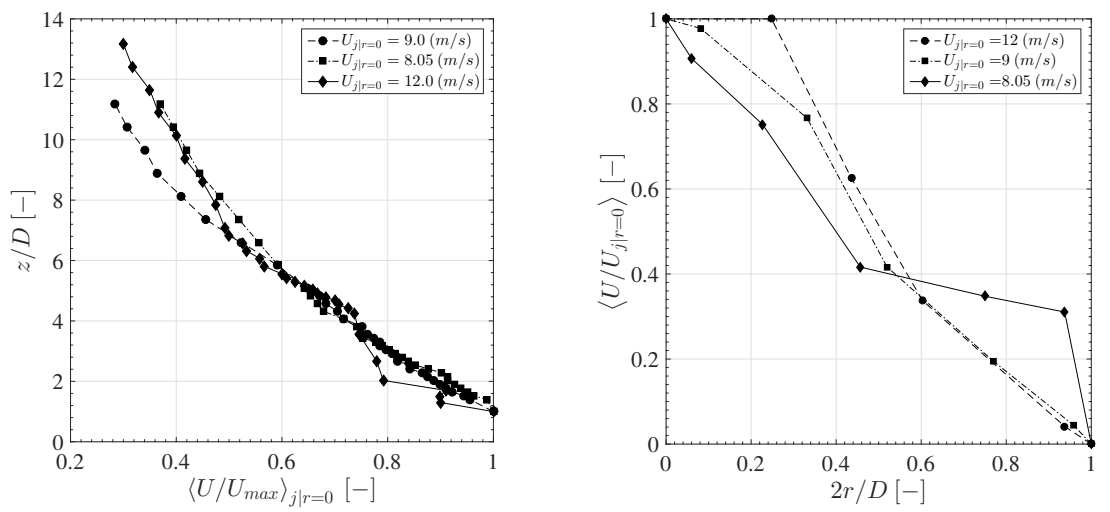

Figure 5: Shown on the left are the time averaged vertical velocities of the jet at the centerline $r=0$ where $r$ is the radial distance from the jet center; And on the right are cross section stream-wise velocities which are measured at $z=0.138 m$ from the jet nozzle exit.

in good agreement with the classical data for round jets (see Albertson et al. [1]). Therefore, it is possible to use the integral model of round jets to characterize and quantify the source condition parameters. This can be done using the following equation for the stream wise velocity of the jet $[26$.

$$
\left\langle U_{j \mid r=0}\right\rangle=7 M_{0}^{1 / 2} z^{-1}
$$

where $M_{0}$ is the initial momentum flux at the source, and $z$ is the vertical distance from the jet virtual origin. In wildfires, $M_{0}$ corresponds to the buoyancydriven momentum at the source which is a function of heat release rate of fire or burning rate of the fuel. Based on equation 11, the time-averaged vertical velocity is proportional to the inverse of distance from the virtual origin $(z)$. This is shown by the log-log plots of the experimental measurements in the quiescent ambient, see figure 6 .

The experimental results in figure 6 clearly demonstrate the length of the potential core is much smaller than the typically observed. This is because of the jet structure in which the air flow is generated by the blowers passes through multiple flexible hoses, and then goes through a flow straightener with honey- 


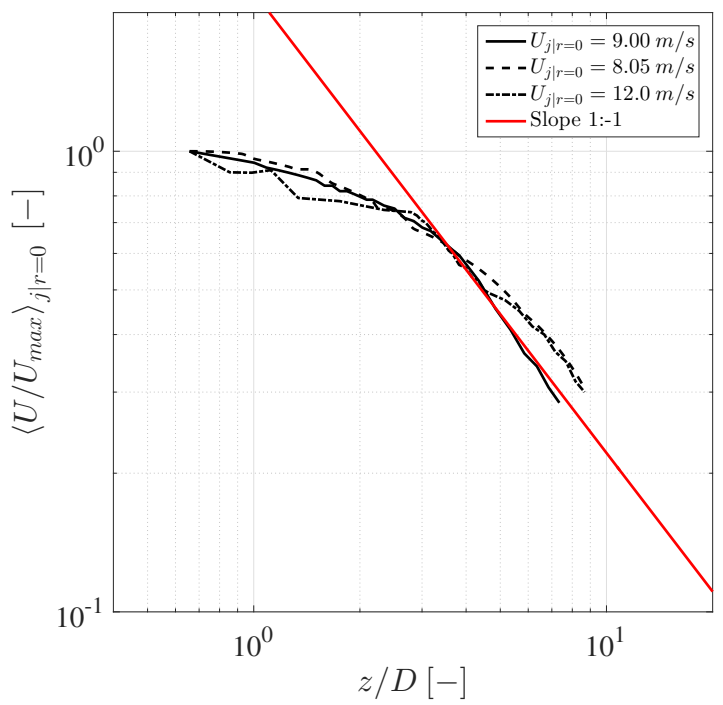

Figure 6: Normalized time averaged vertical velocity of the jet at the centerline.

comb mesh. Hence, the jet outflow is highly turbulent and the jet potential core is eroded in only three diameters downstream. This is much smaller than the $6.2 D$ distance downstream typical of the literature [15, 26], where $D$ is the jet diameter. Also, the predicted variation of the velocity with $z$ in equation 1 is well-confirmed. In fact, having a fully developed turbulent jet flow being entered in a turbulent boundary layer cross-flow ensures that the turbulence effects are factored in and experiments reasonable approximation of real wildfire scenarios. This is important for mathematical modeling of firebrand shower phenomenon as Karimpour and Kaye [23] show that turbulence influences flight statistics of debris.

Different combinations of the wind tunnel boundary layer and jet velocity fields were tested that are analogous to the interaction of a fire plume with the atmospheric boundary layer. To the best of the authors' knowledge, this is the first time the coupled lofting and transport of firebrands has been experimentally examined. Different aspect ratios of rod-like polyurethane model firebrands were 
released at or near the centerline of the jet exit. Each aspect ratio was released approximately 200 times for each combination of the boundary layer and jet velocity. For each set of 200 releases the initial orientation (Euler's angles [19]) was varied, randomly. This provided a significant number of samples (degrees of freedom) for statistical analysis. The entire process of lofting and downwind transport of individual model firebrands are recorded from the side view using a $G C$-PX100 JVC camera with $1920 \times 1080$ resolution at $60 \mathrm{fps}$ (see Figure2. right).

\section{Data acquisition method}

The lofting and downwind transport of the model firebrands was recorded from the side with a camera set to be perpendicular to the centerline of the wind tunnel. An image processing algorithm was developed and implemented in the MATLAB environment in order to capture full trajectories of firebrands along with the maximum rise height and downwind distance. The data acquisition technique is discussed below.

\subsection{Camera calibration}

In order to reach a quasi-steady state, the wind tunnel and the jet were run for $2-3$ minutes, while the camera was being installed on the side view of the test section. Then, for each set and before releasing the model firebrands into the velocity field, a calibration video was taken in which a designated scale was fixed directly over the jet exit into the wind tunnel chamber. The scale was used to calculate the picture ratio of the images and further calibrate (convert) pixels to units of length. Picture ratio is the fraction of known distances between designated black dots on the scale, here in millimeters, to the corresponding number of pixels between them [16]. In addition, optical distortion due to fish-eye effect and any slight camera misalignment was measured for each set of tests utilizing the Omnidirectional Camera Calibration algorithm [42] and found to be less than $\pm 1 \mathrm{~mm}$ for the presented setup. Figure 7 shows a sample 


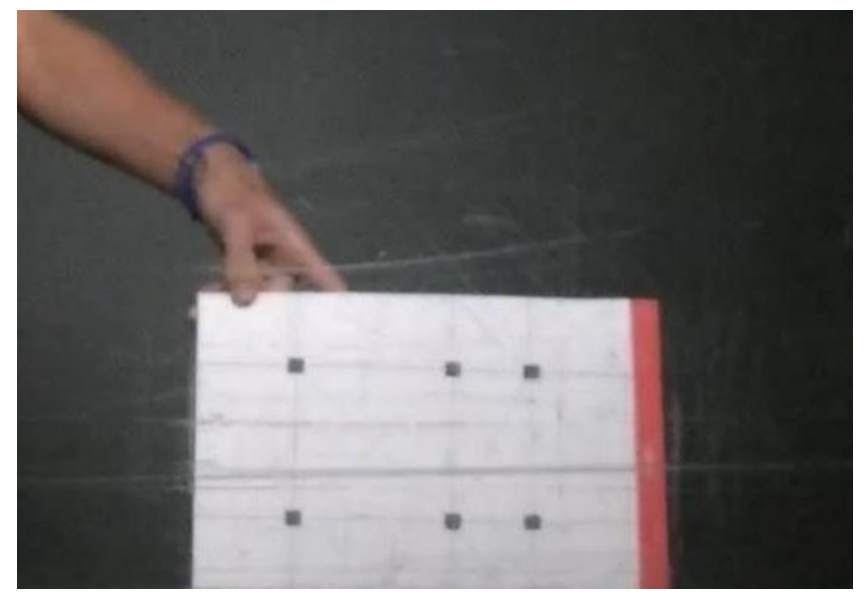

Figure 7: An extracted calibration image magnified on the scale's area in order to present the utilized scale. There are four points on the scale that build a square with sides of $100 \mathrm{~mm}$, and the other two are used to check the calculated picture ratio.

image that is extracted from a calibration video. With regard to accuracy of the imaging technique, one might argue that according to firebrands' transport [38, 48, is a highly stochastic phenomenon and trajectories are 3D with lateral spread which might affect the results. On this note, it should be mentioned that, a small symmetric lateral spread was observed during the post experimental clean up where the locations of the model firebrands on the wind tunnel floor was clustered around the centerline of the tunnel directly downwind of the release location. However, in these set of experiments, since the aerodynamic force of the wind tunnel boundary layer on the Polyurethane made model firebrands is large enough, the observed lateral spread is very small relative to the maximum rise height and the maximum downwind distance. Also, due to the symmetry of the experiments, the lateral spread would have negligible impact on the estimated first order statistics of the results. For future studies, adding another camera and conducting stereophotogrammetry would resolve this minor issue. 


\subsection{Data collection}

After the calibration operation the scene was emptied and videos were recorded of model embers being lofted and transported. Per set, model firebrands were released at least $10 s$ after the camera was turned on. Since the recording was being done with $60 \mathrm{fps}$, that provided enough frames to construct the background image. The firebrand models were then released from approximately the center of the jet with tweezers such that the jet flow field was disturbed as little as possible. For each release operation the initial angles of each model firebrand are varied randomly. Each set of the experiments took between $8-11$ minutes to record the lofting and downwind transport of 200 samples.

\subsection{Image processing}

The videos collected were analyzed using an image processing algorithm that starts with extracting all frames in a video and constructing a background image. The extracted frames are stored as images on an external hard disk due to the high volume of the generated data. Using MATLAB, images are read and converted from RGB to double in gray scale space. This provides more accuracy and flexibility in image analysis. The background image is constructed by averaging over light intensities of at least 100 empty images. On figure 8 left a sample background image is shown versus a normal frame taken from the emptied scene of the test section.
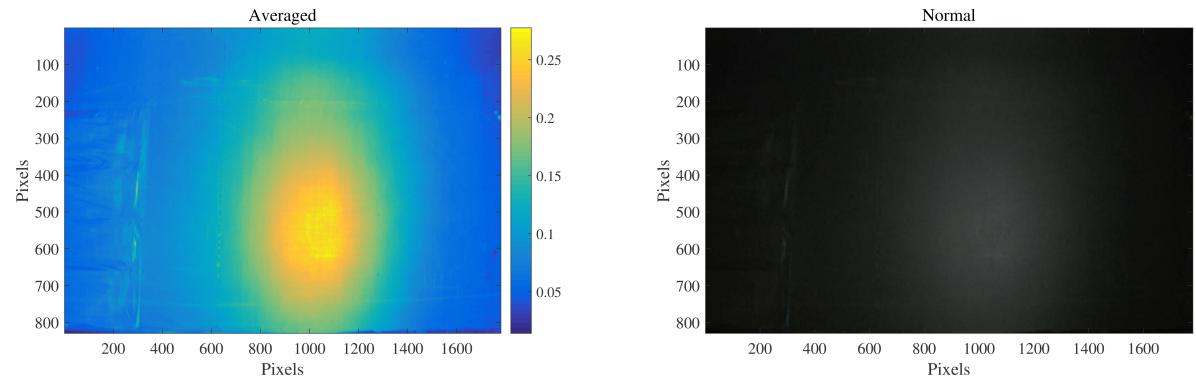

Figure 8: (Left) Color coded background image, with light intensities, constructed by averaging over at least 100 empty frames. (Right) an original (untouched) frame, empty from model firebrands presence at the test section while the velocity field is flowing. 
The background image is subtracted from all frames. This detects the presence of model firebrands in the domain. Yet, the obtained image is not practically useful for calculating the firebrands' position inside the frame. This is due to the general motion of the firebrands during which they are both translating and rotating. Rotation causes model firebrands to emit various range of light intensities such that it is hard to identify all of them by just a global thresholding method; see 17. In order to resolve this issue, background subtracted frames are convolved with a rotationally symmetric low-pass Gaussian filter with a square height matrix of 45 pixels and standard deviation of $\sigma_{g}=5$ as shown in figure 9. Convolution of the Gaussian filter reduces the noise in the image and prepares it for intensity mapping. A sample of the smoothed image is shown on figure 10 (top-right).
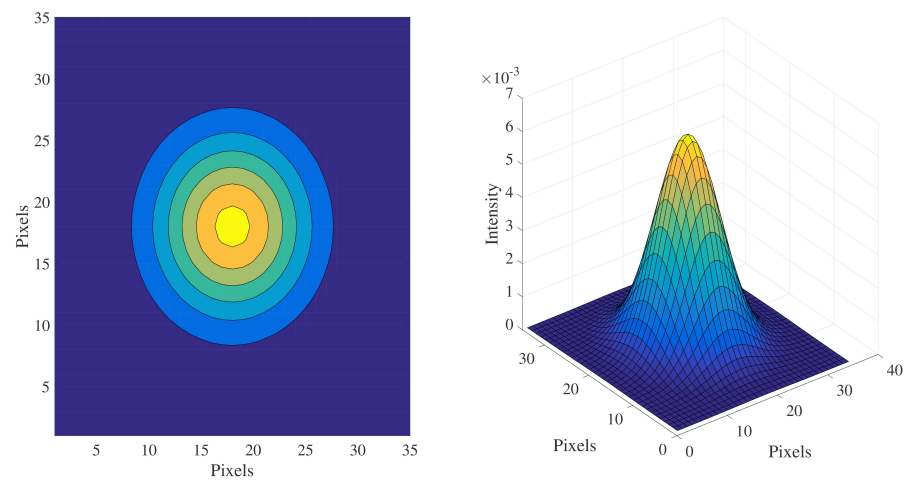

Figure 9: The utilized rotationally symmetric low-pass Gaussian filter that is used for noise reduction. The given height is color coded with the intensity range before noise reduction.

The intensities obtained are then mapped to the range of $0-1$ with high $\Gamma$ function (see [16]) value such that a global thresholding method can be applied. This operation isolates the pixels that the model firebrands occupy in the image; see figure 10-(bottom-left). Ultimately, in order to calculate the centroid coordinates of the identified model firebrand, the intensity stretched image is morphed 

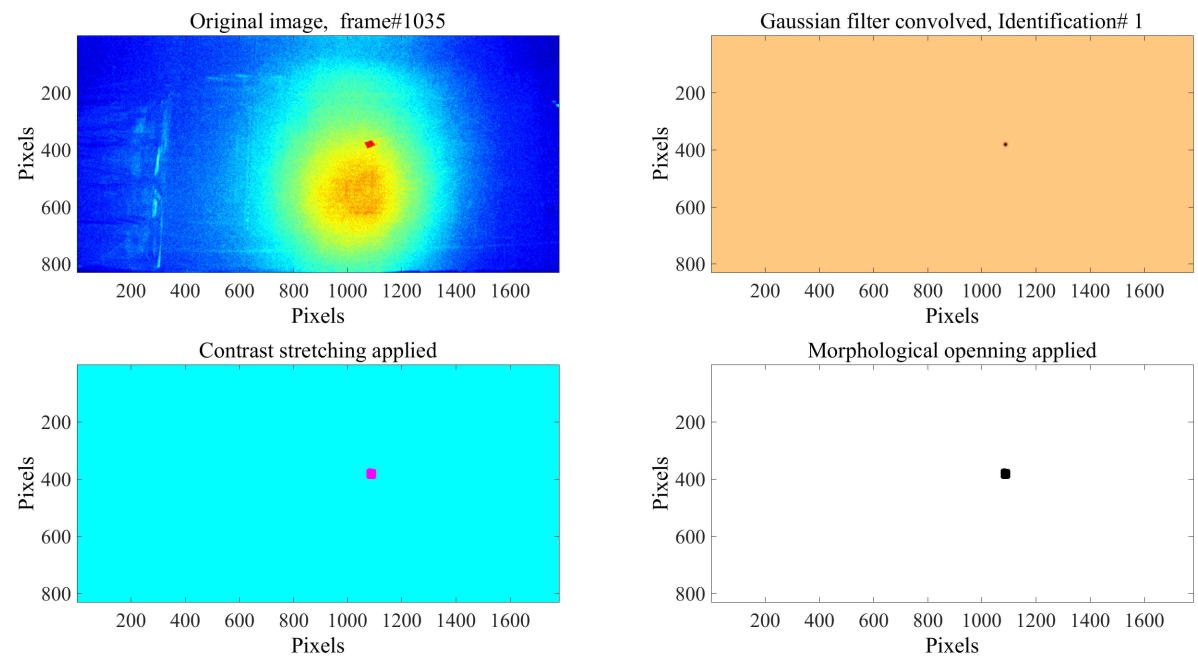

Figure 10: (Top-left) Untouched image from a model firebrand during its flight through the velocity field. (Top-right) Shown is the result of background subtraction and the Guassian filter convolution on the original image. The image with mapped intensity along with the morphologically opened image are shown at the bottom-left and bottom right, respectively. The dot in the middle is a model firebrand with $\eta=1$ that is identified in frame 1035 of the video, that is $17 s$ after the release operation has started for this set of experiments.

with the cylindrical (in cases with $\eta=4$ or 6 ) and square (in cases with $\eta=1$ ) structuring elements. This process is repeated over all extracted images and results in resolving full 2D trajectories of the model firebrands during lofting and downwind transport; see figure 10 (bottom-right). Sample trajectories resulting from applying this algorithm on a sample video are shown in Figure 11.

Two recurring problems were observed among the obtained trajectories. First, some of the trajectories do not include the exact landing location. This is primarily due to the low light intensity near the floor which makes the algorithm susceptible to miss the objects as they pass through the camera frame. This issue is resolved by extrapolation of the obtained trajectories to the elevation of $z=0$. The extrapolation is conducted by converting the units from millimeter to meter such that the remaining distance to the ground, i.e. extrapolation domain, is normalized between $0-1$. This reduces the extrapolation error, since 


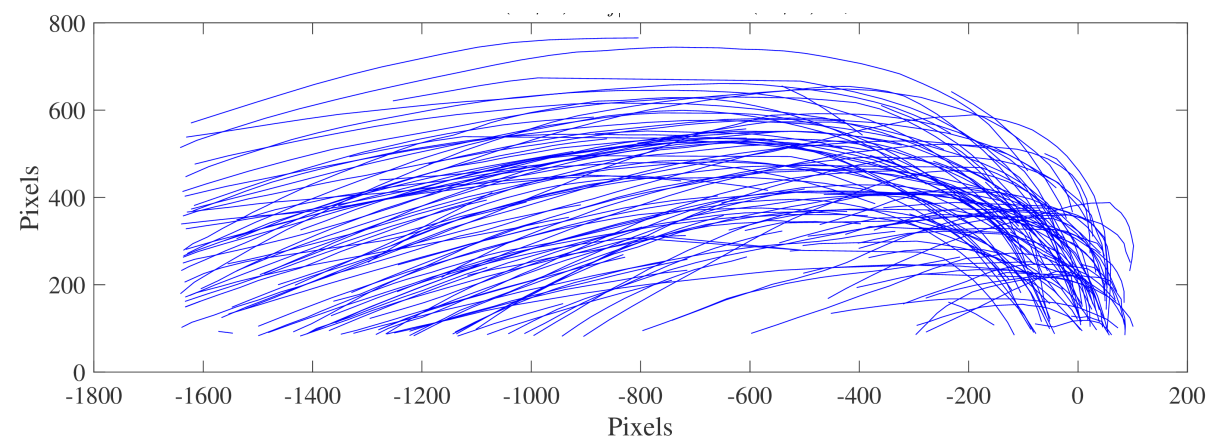

Figure 11: Calculated trajectories of the model firebrands with aspect ratio $\eta=1$ in an experimental case where the boundary layer reference velocity was $U_{0}=2.23 \mathrm{~m} / \mathrm{s}$ (from right to left), and the jet centerline velocity at the exit (point $[0,0]$ ) was $8.05 \mathrm{~m} / \mathrm{s}$.

the last three points on the trajectory are chosen as the training set for finding the corresponding point (landing location) on the ground. The same method is applied to the upstream part of the trajectory where the model firebrands lift off through the jet.

Second, some flights resulted in incomplete or partially identified trajectories. This is caused by either the model firebrands leaving the frame of analysis due to high lofting velocity or low intensity of light during the flight. The later issue is resolved by the data storage technique that is used in the algorithm. This method assigns a tag to each detected object and saves that with the position coordinates. In this way if a trajectory is partially recognized (say if the model firebrand is lofted out of the frame) the subsequent parts can be combined together. The former issue is solved by using characteristics of the completed trajectories. As is evident in figure 12 full trajectories have a pattern if one calculates the adjacent difference in the rise height at each point, namely $\Delta y=y_{i}-y_{i-1}$. If the resulting vector (for each model firebrand trajectory) is drawn against the order by which the location coordinates are recognized (i.e. $i)$, the pattern has three distinct parts. The first part where $\Delta y$ is decreasing can be associated with the lofting part, the section with effectively zero slope 

tories with a corrupted tag, they are eliminated from calculations.

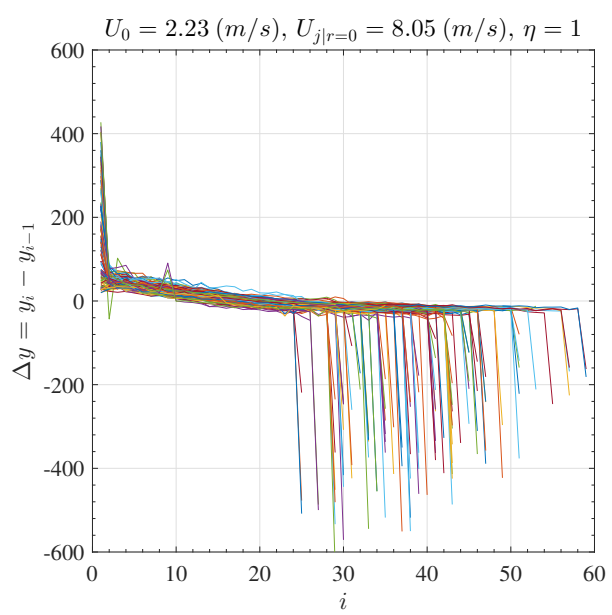

can be attributed to the lofting to downwind transport transition, and finally as $\Delta y$ decreases again the landing part has been started. Even the shortest complete trajectories have more than 10 identified points in their position coordinates vectors. Incomplete and partial trajectories do not follow such patterns or simply don't have enough points; This has been confirmed through many trials within the algorithm (figure 12 right). Further, trajectories in which more than $5 \%$ of the identified points are off this pattern are assigned as incomplete. Lastly, if there is no corresponding partial or incomplete path found for trajec-

Figure 12: The identified pattern in complete trajectories by calculating adjacent differencing of the rise height (left). On the right shown are samples of the incomplete or partially complete trajectories that do not follow the same pattern as the completed ones.

331

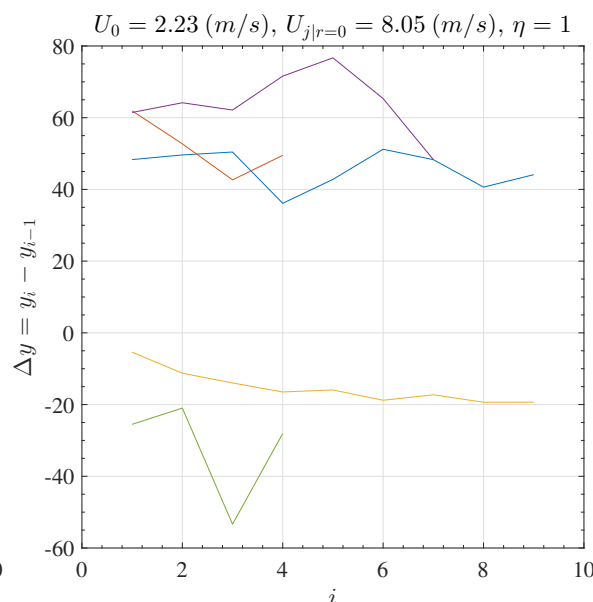

\section{Results}

Tests were run for all 27 combinations of three wind speeds, jet speeds, and firebrand aspect ratios. Approximately 200 releases were done for each parameter combination, though some of the model firebrands are missed during the image processing procedure. Therefore, the reported DOFs are not equal to 

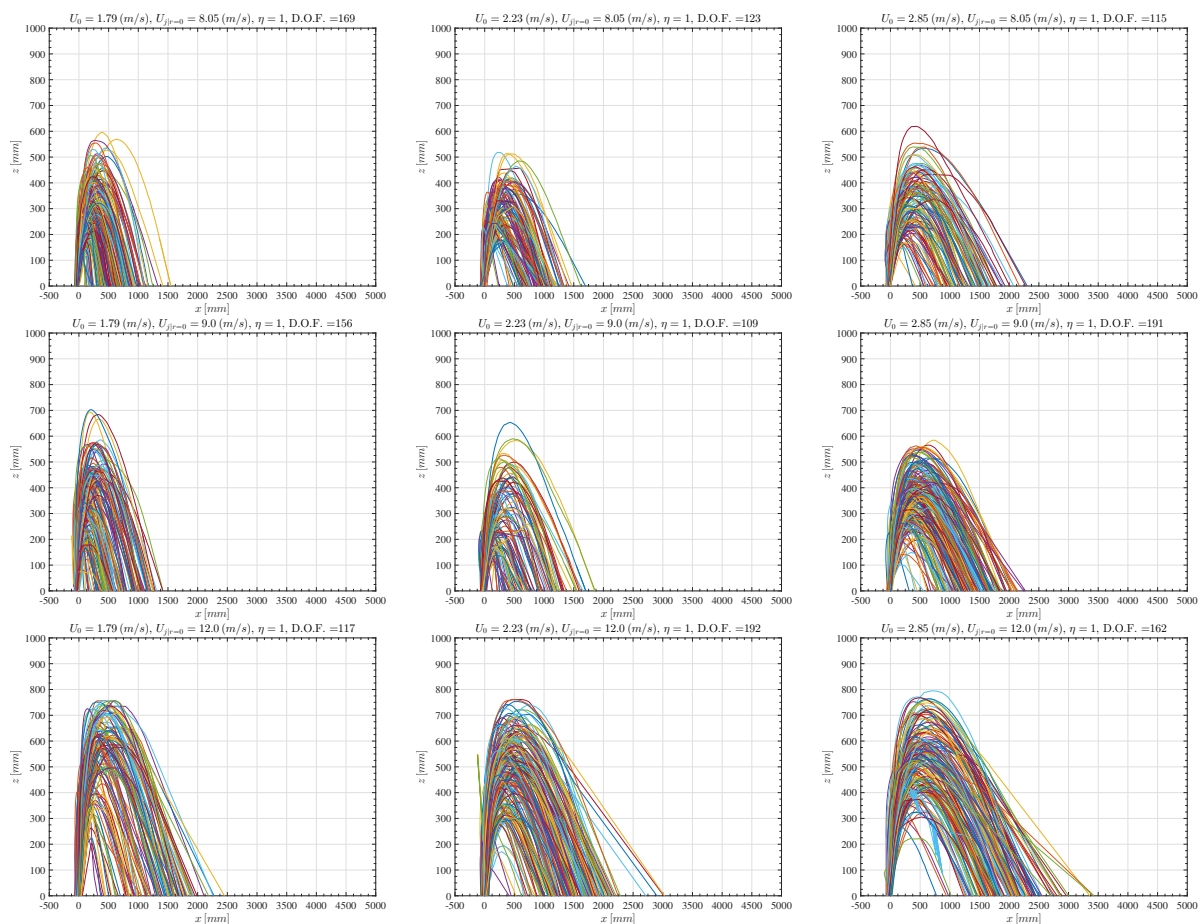

Figure 13: Obtained trajectories from the image processing analysis. Presented data are trajectories of the cubic firebrand models with longitudinal and side aspect ratio of one, i.e. $\eta=\eta_{s}=1$. From left to right, the reference velocities of the wind tunnel boundary layer are $1.79,2.23$, and $2.85(\mathrm{~m} / \mathrm{s})$, and from top to bottom, the jet centerline velocities are 8.05, 9.0, and $12(\mathrm{~m} / \mathrm{s})$.

The captured trajectories are presented in figures 13 15. Generally, the re- 

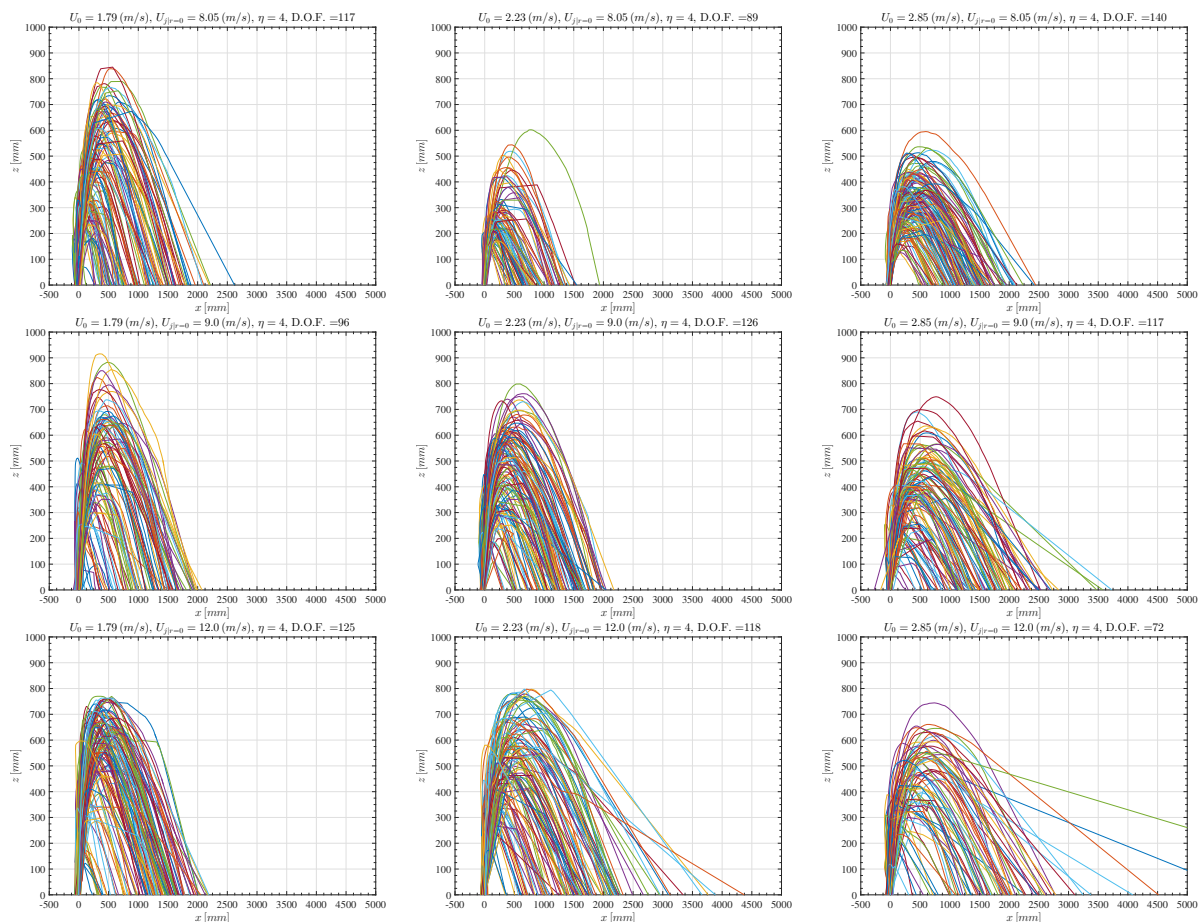

Figure 14: Obtained trajectories from the image processing analysis. Presented data are trajectories of the cylindrical firebrand models with longitudinal aspect ratio $\eta=4$. From left to right, the reference velocities of the wind tunnel boundary layer are 1.79, 2.23, and $2.85(\mathrm{~m} / \mathrm{s})$, and from top to bottom, the jet centerline velocities are 8.05, 9.0, and $12(\mathrm{~m} / \mathrm{s})$.

sults corroborate the physical expectations, namely, by increasing the boundary layer reference velocity $\left(U_{0}\right)$ for a given jet exit velocity $\left(U_{j \mid r=0}\right)$ and aspect ratio $(\eta)$, model firebrands tend to have lower rise height and land further downwind.

Characteristic lengths can be extracted from each trajectory, which include the maximum rise height $\left(z_{\max }\right)$, and the maximum downwind distance $\left(x_{l}\right)$. Since the velocity field of the fire plume is modeled as a jet, the dominant length scale for the jet in a non-uniform boundary layer cross flow is the jet momentum length $z_{m}$; see Tohidi and Kaye [51. The momentum length represents the vertical distance at which a pure point source jet will have a mean vertical velocity equal to the mean horizontal velocity of the boundary layer [51]. In wind tunnel 

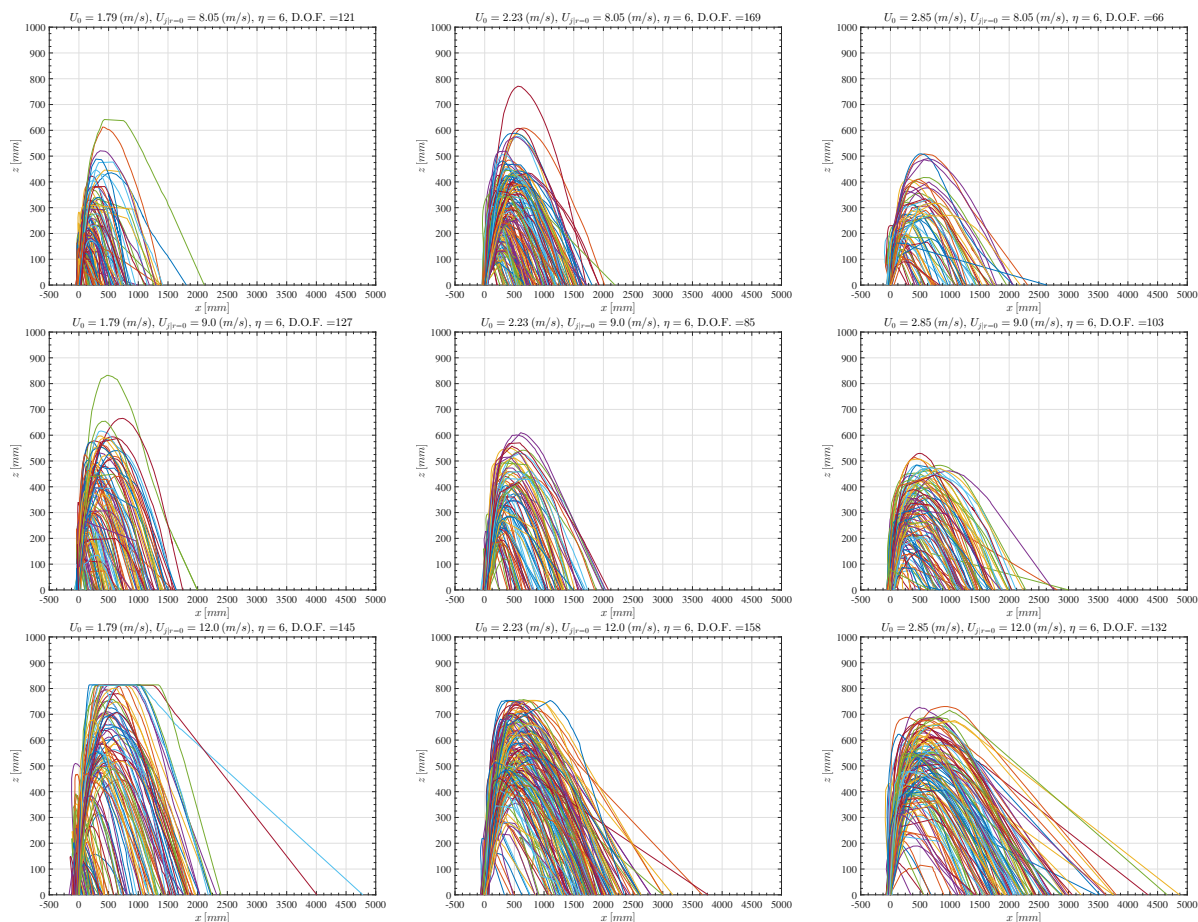

Figure 15: Obtained trajectories from the image processing analysis. Presented data are trajectories of the cylindrical firebrand models with longitudinal aspect ratio $\eta=6$. From left to right, the reference velocities of the wind tunnel boundary layer are 1.79, 2.23, and $2.85(\mathrm{~m} / \mathrm{s})$, and from top to bottom, the jet centerline velocities are 8.05, 9.0, and $12(\mathrm{~m} / \mathrm{s})$.

experiments, this length scale can be easily obtained by finding the height of the intersection point between the vertical (stream-wise) velocity profile of the jet at its centerline and the horizontal velocity of the boundary layer. In the case of a real fire plume the analogous length scale is the buoyancy length, $z_{f}[51]$. The buoyancy length is the distance from source at which a pure point source plume will have a mean vertical velocity equal to the mean horizontal velocity of the cross-flow boundary layer; For more details on the significance of $z_{m}$ and $z_{f}$ on wildfires' flow field see Tohidi and Kaye [51. As $z_{m}$ is the dominant length scale of the jet in non-uniform boundary layer cross-flow, it is used to normalize the maximum rise height and the maximum downwind distance. These data are summarized in figures 16 17 where the empirical (kernel) probability den- 
sity functions of each parameter are shown for the tests under the same initial conditions.
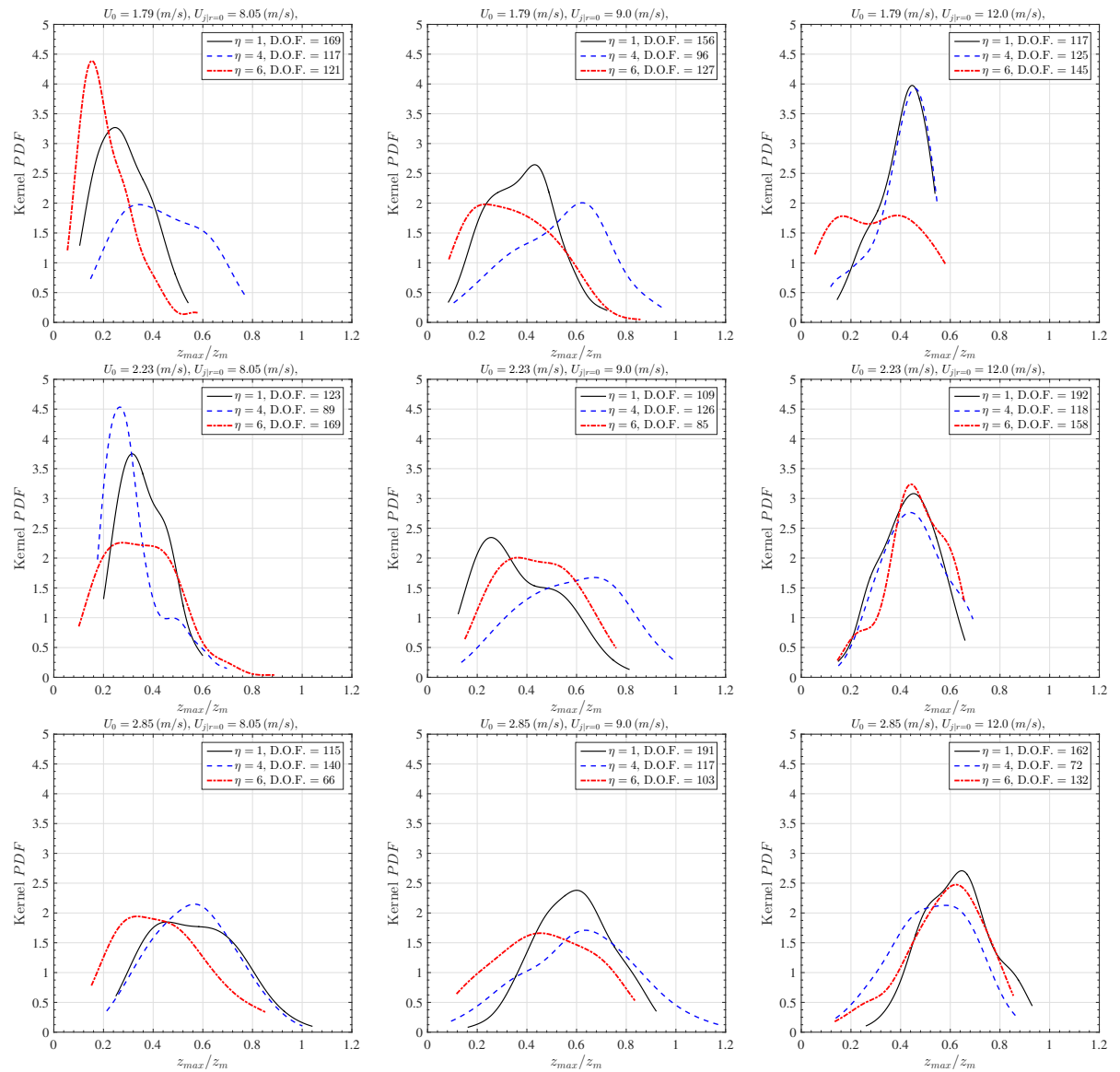

Figure 16: Shown are the empirical (kernel) probability density functions (PDF) of $z_{\max } / z_{m}$ for different aspect ratios, under various initial conditions of the wind tunnel experiments. From left to right, $U_{0}$ is constant while $U_{j \mid r=0}$ is increasing. From top to bottom, $U_{0}$ is increasing and $U_{j \mid r=0}$ is constant.

Figure 16illustrates variations of the normalized maximum rise height of the model firebrands with different aspect ratios subjected to various initial conditions of the wind tunnel experiments. As can be seen, for low $U_{0}$ as $U_{j \mid r=0}$ increases the mean increases as well as its standard deviation. The foregoing 

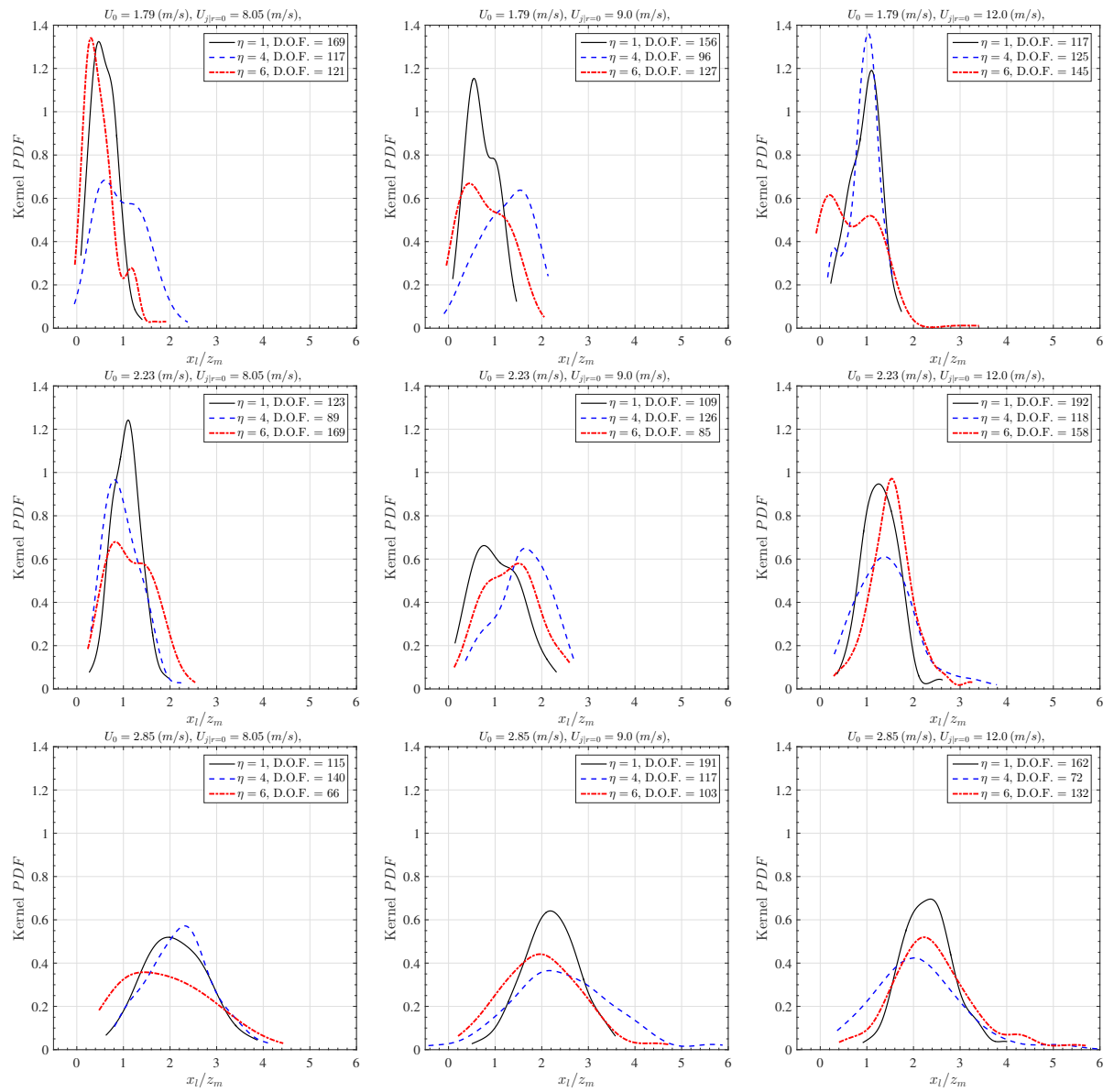

Figure 17: Shown are the empirical (kernel) probability density functions (PDF) of $x_{l} / z_{m}$ for different aspect ratios, under various initial conditions of the wind tunnel experiments. From left to right, $U_{0}$ is constant while $U_{j \mid r=0}$ is increasing. From top to bottom, $U_{0}$ is increasing and $U_{j \mid r=0}$ is constant. 

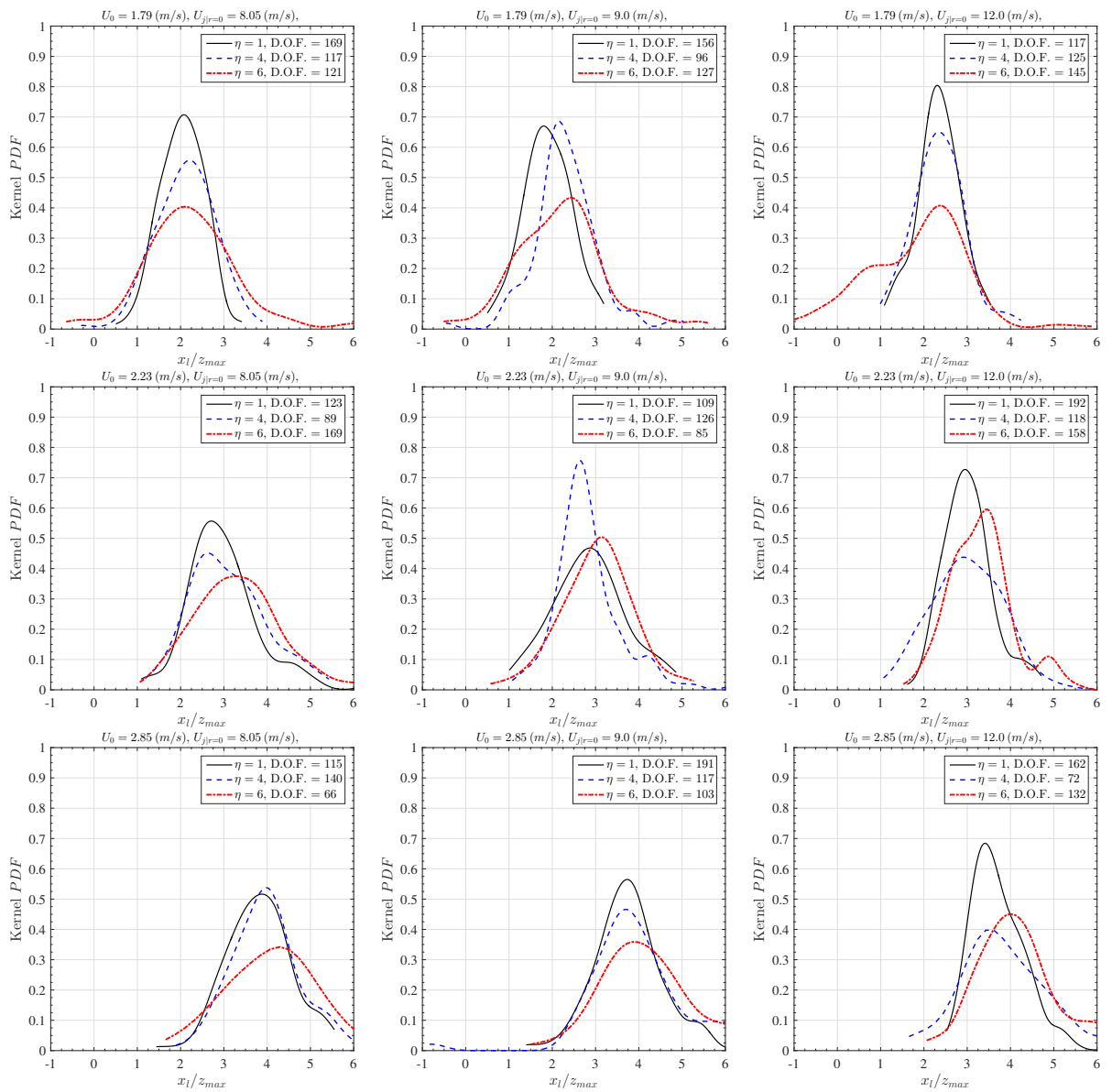

Figure 18: Shown are the empirical (kernel) probability density functions (PDF) of $x_{l} / z_{\max }$ for different aspect ratios, under various initial conditions of the wind tunnel experiments. From left to right, $U_{0}$ is constant while $U_{j \mid r=0}$ is increasing. From top to bottom, $U_{0}$ is increasing and $U_{j \mid r=0}$ is constant.

As for of the normalized maximum downwind distance shown in figure 17 in the low wind conditions, i.e. $U_{0}=1.79(\mathrm{~m} / \mathrm{s})$, increasing the jet velocity increases the mean. However for firebrands with $\eta=1,4$ it does not significantly increase the standard deviation. Quite the contrary, for the largest aspect ratio, increasing the jet velocity not only increases the mean and standard deviation of $x_{l} / z_{m}$ but also leads to bimodal distribution of the normalized landing location in some cases. The bimodal ground-distribution of firebrands has been reported 
previously by Sardoy et al. 39. For moderate to high wind conditions of the experiment, increasing the jet velocity causes the model firebrands to loft higher and subsequently travel further downwind.

The most striking result emerges when the maximum downwind distance $\left(x_{l}\right)$ is normalized by the maximum lofting height $\left(z_{\max }\right)$. Despite different degrees of freedom for each aspect ratio, PDFs of $x_{l} / z_{\max }$ are very similar. In fact for a given velocity field, PDFs of different aspect ratios collapse on each other. As can be see on figure 18 , for a given boundary layer velocity, increasing the jet speed does not result in a significant change in the mean and standard deviation. However, for a given jet speed, an increase in the boundary layer velocity increases the mean and standard deviation. This is further shown by figure 19 where the maximum landing location $\left(x_{l}\right)$ is plotted against the maximum rise height $\left(z_{\max }\right)$ for all released model firebrands during the wind tunnel experiments. Clearly, there is a strong positive correlation between $x_{l}$ and $z_{\max }$.

Similarity of $x_{l} / z_{\max }$ PDFs and the observed positive correlation signify the fact that there is a relationship, even though geometric, between the maximum rise height and the maximum downwind distance of a firebrand traveling through a given velocity field. Therefore, previous studies [52, 20, 41, 40, 39, 13, 43] in which firebrands or debris are released from a fixed arbitrary height should be careful about the justification of the chosen release heights as the maximum rise height of firebrands will have significant influence on the modeled flight distance. Another implication of these results is that for mathematical modeling of the lofting and downwind transport of firebrands/debris, lofting cannot be decoupled from the transport through the atmospheric boundary layer.

Further insight may be obtained by summarizing the experimental parameters and conditions in terms of non-dimensional groups embedded in the physics 


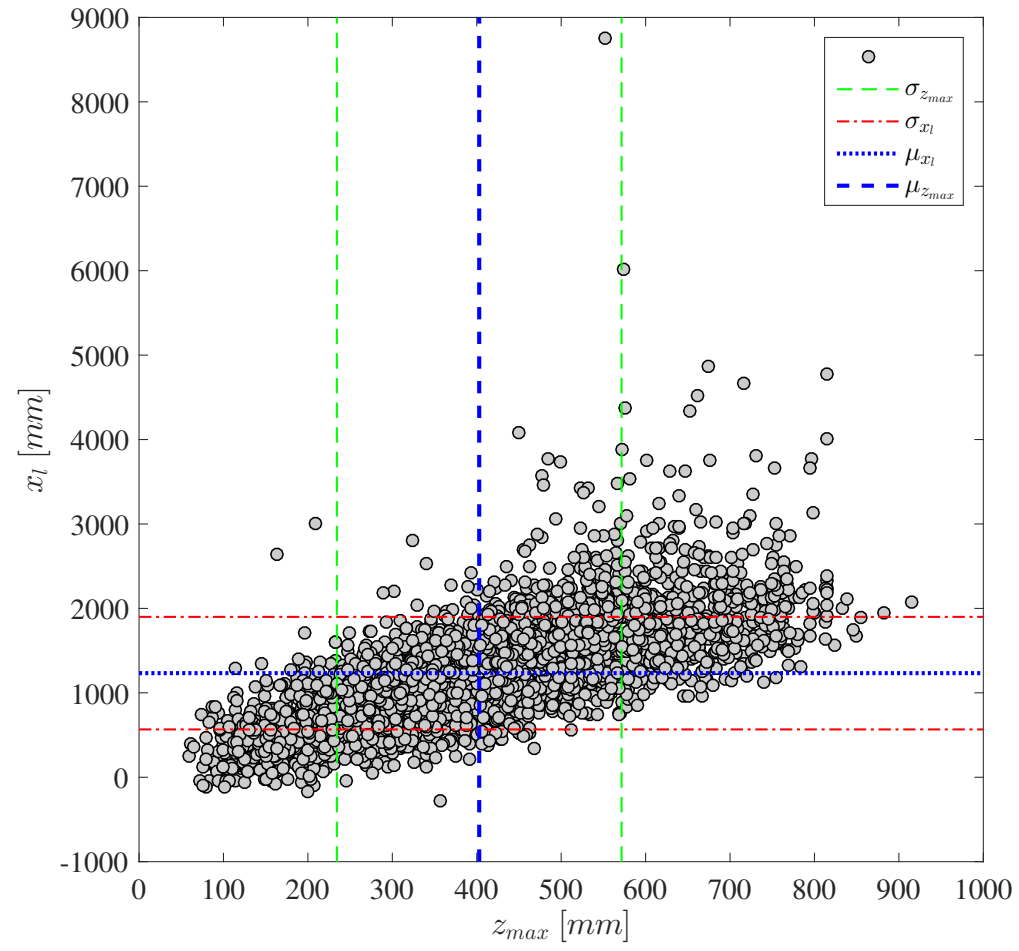

Figure 19: Scatter plot of the maximum landing location $\left(x_{l}\right)$ against the maximum rise height $\left(z_{\max }\right)$ for all resolved trajectories during the experiments. Here, $\mu$ denotes the average and $\sigma$ shows the standard deviation.

of the problem. The controlling non-dimensional groups are

$$
\frac{U_{0}}{U_{j \mid r=0}}, \eta, \text { and } \Omega=\frac{\rho_{a} U_{0}^{2}}{\rho_{s} g L_{z}}
$$

where, as mentioned before, $U_{0} / U_{j \mid r=0}$ is the ratio of the boundary layer reference velocity to the jet centerline velocity at the exit, and $\eta$ is the aspect ratio of the model firebrands. The additional parameter $\Omega$ is the Tachikawa number which is the ratio of the aerodynamic forces to the gravity force [22]. Here, $\Omega$ is calculated based on the largest projected area of the firebrands (the largest dimension, i.e. $\left.L_{z}\right)$. Respectively, $\rho_{a}$ and $\rho_{s}$ are density of the ambient air and the model firebrands. Since the combustion effects are decoupled from the 
lofting and downwind transport in these experiments, $\rho_{s}$ is constant. In fact, the time scale for a given flight in our experiments is small (approximately $1-2$ seconds) such that any mass loss, were the model firebrands combusting, would be very small and have negligible impact on the flight dynamics. Given that the important variables $x_{l}, z_{\max }$, and the jet momentum length scale $\left(z_{m}\right)$ can be obtained from the measured velocity profiles, the results can be presented as

$$
\left(\frac{x_{l}}{z_{m}}, \frac{z_{\max }}{z_{m}}\right)=\Pi\left(\frac{U_{0}}{U_{j \mid r=0}}, \eta, \Omega\right) .
$$

Figure 20 shows variations of the mean $(\mu)$ and standard deviation $(\sigma)$ of $x_{l} / z_{m}$, $z_{\max } / z_{m}$, and $x_{l} / z_{\max }$ against $\Omega$. As shown, by increasing $\Omega$, i.e. greater boundary layer reference velocity or smaller $L_{z}$, the mean of $x_{l} / z_{m}$ and $x_{l} / z_{\max }$ increases. Also, the greater the aspect ratio, the greater the rate of increase in the mean and standard deviation of $x_{l} / z_{m}$, and $x_{l} / z_{\max }$; see figure 20 top \& bottom. Also, the results suggest that the sensitivity of $x_{l}$ to the change in the velocity field is higher for larger aspect ratios. However, the mean and standard deviation of the normalized rise height $\left(z_{\max } / z_{m}\right)$ tend to increase with very mild slopes as $\Omega$ increases, see figure 20 middle.

The distribution of the mean and standard deviation of $x_{l} / z_{m}$ and $z_{\max } / z_{m}$ within the parameter space of the experiments, given in equation 2 , are presented by interpolated surfaces between the experimental results for each aspect ratio. These results are shown in figures 2123 The results of $\eta=1 \& 6$ satisfy the physical expectations, in that for a given aspect ratio and boundary layer reference velocity $(\Omega=$ const.), increasing the velocity ratio (decreasing the jet velocity) decreases the maximum rise height and subsequently the maximum downwind distance. Also, for a given wind velocity ratio $\left(U_{j \mid r=0}=\right.$ const. $)$ increasing $\Omega$ increases the applied aerodynamic forces on the brands and leads to greater flight distances. Alternatively, results of $\eta=1 \& 6$ can be interpreted as traveling along characteristic curves (gradients of the contours) from any point on the parameter space leads to increasing aerodynamic forces either due to jet 
or boundary layer and would increase the rise height or maximum downwind distance, respectively. However, results of model firebrands with aspect ratio $\eta=4$ do not follow this trend, and there are lot of variability in the statistics of their flight as can be seen on figure 22. Nevertheless, the characteristic curves of the contour plots for different aspect ratios seem to follow the same trend.

A summary of the wind tunnel experimental results are given in table 2 . The experimental data base can be accessed online by contacting the lead author.

\section{Conclusions}

Results of the most extensive set of large scale wind tunnel experiments of the lofting and downwind transport of model firebrands are presented. The data serves as an invaluable resource for not only firebrand transport studies but also for the flight of rod-like debris/brands through other velocity fields generated by extreme events such as hurricanes and storms. Full trajectories of the model firebrands were captured utilizing the developed image processing algorithm. From the captured trajectories, the maximum rise height and landing location of the model firebrands were obtained. It is shown that, given the velocity field, the maximum landing locations normalized with the maximum rise heights $\left(x_{l} / z_{\max }\right)$ have similar probability density functions (PDF) regardless of the firebrands' aspect ratio. This implies that, in modeling firebrand shower and the subsequent fire spotting phenomenon, unlike some previous studies, the lofting and downwind transport of the firebrands cannot be decoupled, since the maximum rise height significantly affects the ground distribution of firebrands.

The experimental results are presented in terms of the non-dimensional parameters, i.e. velocity ratio, aspect ratio, and Tachikawa number $(\Omega)$. It is shown that there is a strong positive correlation between the Tachikawa number and the mean and standard deviation of the landing locations, normalized with $z_{m}$ and $z_{\max }$. However, a very weak correlation between $\Omega$ and the statis- 
Table 2: Summary of the wind tunnel experiments' results. Here, $\mu$ denotes the mean and $\sigma$ shows the standard deviation of the data.

\begin{tabular}{|c|c|c|c|c|c|c|c|c|}
\hline$U_{0}(\mathrm{~m} / \mathrm{s})$ & $U_{j \mid r=0}(\mathrm{~m} / \mathrm{s})$ & $\eta$ & $\mu_{x_{l}}(m m)$ & $\sigma_{x_{l}}(m m)$ & $\left(\frac{\sigma}{\mu}\right)_{x_{l}}$ & $\mu_{z_{\max }}(m m)$ & $\sigma_{z_{\max }}(m m)$ & $\left(\frac{\sigma}{\mu}\right)_{z}$ \\
\hline \multirow{9}{*}{1.79} & \multirow{3}{*}{9.00} & 1 & 686.55 & 309.96 & 0.45148 & 363.17 & 130.67 & 0.3598 \\
\hline & & 4 & 1190.3 & 520.04 & 0.43688 & 513.17 & 186.99 & 0.36439 \\
\hline & & 6 & 757.4 & 481.36 & 0.63554 & 332.03 & 158.2 & 0.47645 \\
\hline & \multirow{3}{*}{8.05} & 1 & 635.51 & 286.95 & 0.45153 & 308.09 & 114.38 & 0.37124 \\
\hline & & 4 & 1045.8 & 544.23 & 0.5204 & 477.89 & 176.62 & 0.36958 \\
\hline & & 6 & 551.56 & 393.15 & 0.7128 & 233.34 & 113.46 & 0.48625 \\
\hline & \multirow{3}{*}{12.00} & 1 & 1339.3 & 463.26 & 0.34589 & 564.53 & 139.03 & 0.24627 \\
\hline & & 4 & 1307.7 & 464.54 & 0.35523 & 559.45 & 159.25 & 0.28465 \\
\hline & & 6 & 970.71 & 832.62 & 0.85774 & 438.83 & 228.86 & 0.52152 \\
\hline \multirow{9}{*}{2.23} & \multirow{3}{*}{9.00} & 1 & 841.86 & 407.86 & 0.48447 & 297.2 & 129.52 & 0.43579 \\
\hline & & 4 & 1280.9 & 454.2 & 0.3546 & 463.64 & 156.96 & 0.33855 \\
\hline & & 6 & 1076.8 & 469.5 & 0.43602 & 353.11 & 122.55 & 0.34706 \\
\hline & \multirow{3}{*}{8.05} & 1 & 913.75 & 266.59 & 0.29176 & 311.13 & 80.788 & 0.25966 \\
\hline & & 4 & 842.21 & 332.98 & 0.39536 & 276.5 & 98.743 & 0.35712 \\
\hline & & 6 & 996.58 & 428.5 & 0.42997 & 301.43 & 125.65 & 0.41683 \\
\hline & \multirow{3}{*}{12.00} & 1 & 1489.4 & 448.77 & 0.30132 & 496.92 & 132.4 & 0.26644 \\
\hline & & 4 & 1651.2 & 763.61 & 0.46245 & 527.31 & 146.77 & 0.27834 \\
\hline & & 6 & 1766.7 & 565.31 & 0.31998 & 532.51 & 138.09 & 0.25933 \\
\hline \multirow{9}{*}{2.85} & \multirow{3}{*}{9.00} & 1 & 1386.4 & 374.92 & 0.27043 & 373.04 & 96.265 & 0.25806 \\
\hline & & 4 & 1556.3 & 705.6 & 0.45338 & 390.62 & 140.88 & 0.36065 \\
\hline & & 6 & 1282.2 & 549.97 & 0.42892 & 296.63 & 122.84 & 0.41412 \\
\hline & \multirow{3}{*}{8.05} & 1 & 1167.3 & 397.23 & 0.34031 & 308.28 & 106.23 & 0.34458 \\
\hline & & 4 & 1315.5 & 412.66 & 0.31368 & 332.58 & 96.826 & 0.29114 \\
\hline & & 6 & 1146.1 & 555.42 & 0.48461 & 262.17 & 104.28 & 0.39776 \\
\hline & \multirow{3}{*}{12.00} & 1 & 1980.5 & 474.42 & 0.23955 & 535.39 & 117.58 & 0.21961 \\
\hline & & 4 & 1953.1 & 1243.9 & 0.63692 & 448.98 & 134.06 & 0.29859 \\
\hline & & 6 & 2085.7 & 779.35 & 0.37366 & 491.38 & 134.84 & 0.27442 \\
\hline
\end{tabular}

470

tics of the rise height normalized with $z_{m}$ was found. The results show high variability indicating that lofting and downwind transport of non-combusting firebrands is likely extremely sensitive to the initial conditions of the release and to temporal and spatial variations of the ambient velocity field. Also, it is shown that the larger the aspect ratio of firebrands, the more sensitive their landing locations are to the change in the velocity field, particularly the applied aerodynamic force. 
The results presented herein are not directly applicable to full scale wildfires. However, the observations and data have significant implications for the modeling of firebrand transport in wildfires and the resulting predictions of spot fire risk. First, even under controlled laboratory conditions, there is significant variability and, therefore, uncertainty in firebrand flight trajectories. Second, the flight distance is strongly dependent on the maximum lofting height and, therefore, the lofting and wind driven transport components of firebrand flight cannot be decoupled and the entire wind field must be modeled. Third, the shape of the firebrands is important. The model firebrands were observed to rotate and, at times, move transverse to the wind direction. Therefore, lift forces are important in modeling firebrand flight and simplifying assumptions that treat firebrands as compact debris with no rotational inertia or lift forces are likely to miss significant flight dynamics and may under-predict the potential flight distance of a given firebrand. Finally, firebrand flight models need to be run for a large number of flights in order to establish a statistically significant understanding of the probability distribution of firebrand landing locations and resulting spot fire risk. Therefore, firebrand transport models should include a coupled fire plume and atmospheric boundary layer three dimensional wind field, appropriately shaped fire brands, and a firebrand aerodynamic force model that accounts for drag, lift, and rotational forces and moments. Model approaches that satisfy these criteria can then be validated using the experimental results presented herein prior to being applied to full-scale fires.

\section{Acknowledgment}

The authors would like to thank Meredith Frye and Alexandra Maass for their assistance with running the experiments and Danny Metz, Scott Black and Sam Biemann for their help in building the test rig. This material is based upon work supported by the National Science Foundation under Grant no.1200560. Any opinions, findings, and conclusions or recommendations expressed in the 
506 material are those of the author and do not necessarily reflect the views of the $507 \mathrm{NSF}$ 

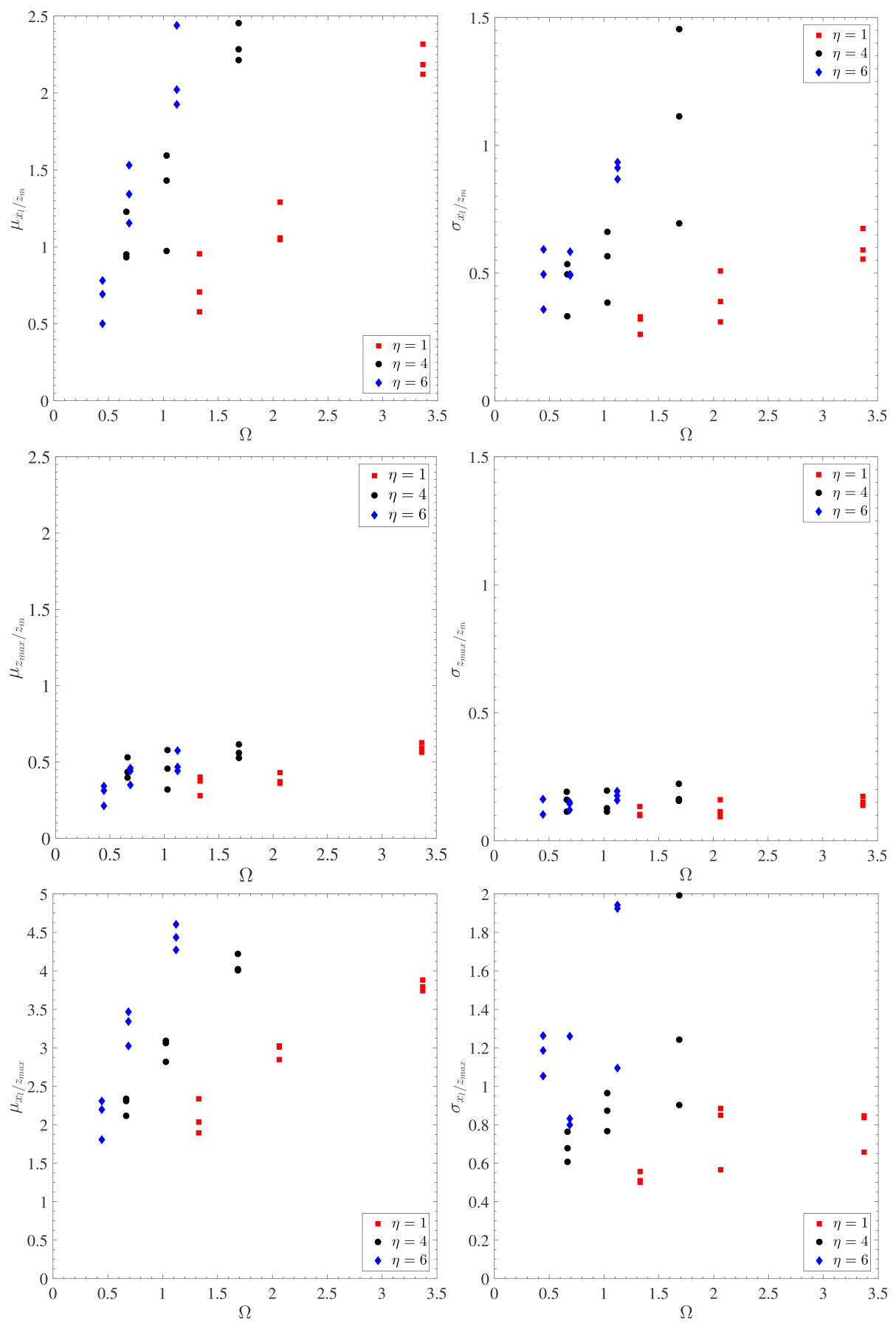

Figure 20: From top to bottom, illustrated are variations of the mean (left) and standard deviation (right) of $x_{l} / z_{m}, z_{\max } / z_{m}$, and $x_{l} / z_{\max }$ of model firebrands with respect to $\Omega$. 

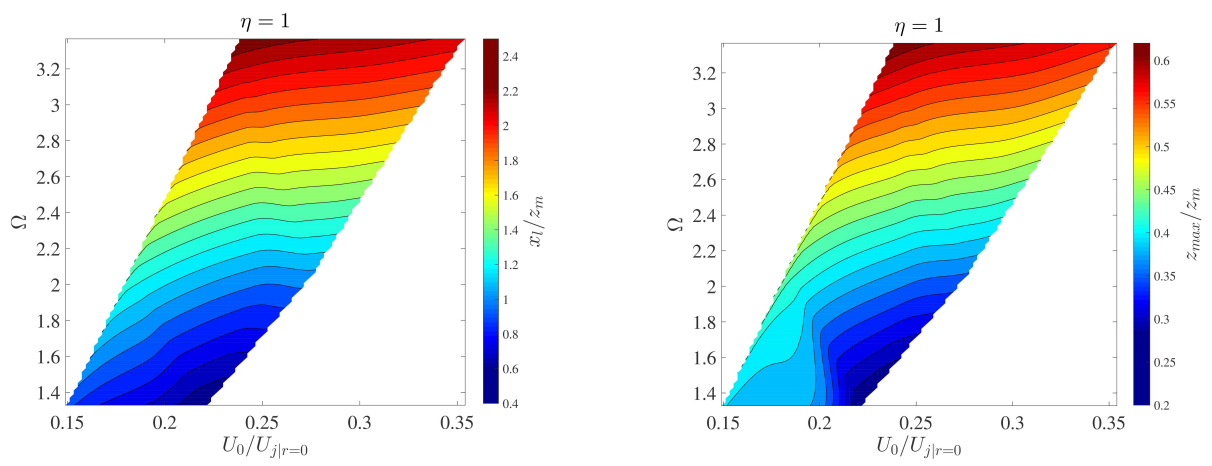

Figure 21: Contour plots of the mean $x_{l} / z_{m}$ (left) and the mean $z_{\max } / z_{m}$ (right) for model firebrands with aspect ratio $\eta=1$ within the parameter space of $\Omega-U_{0} / U_{j \mid r=0}$
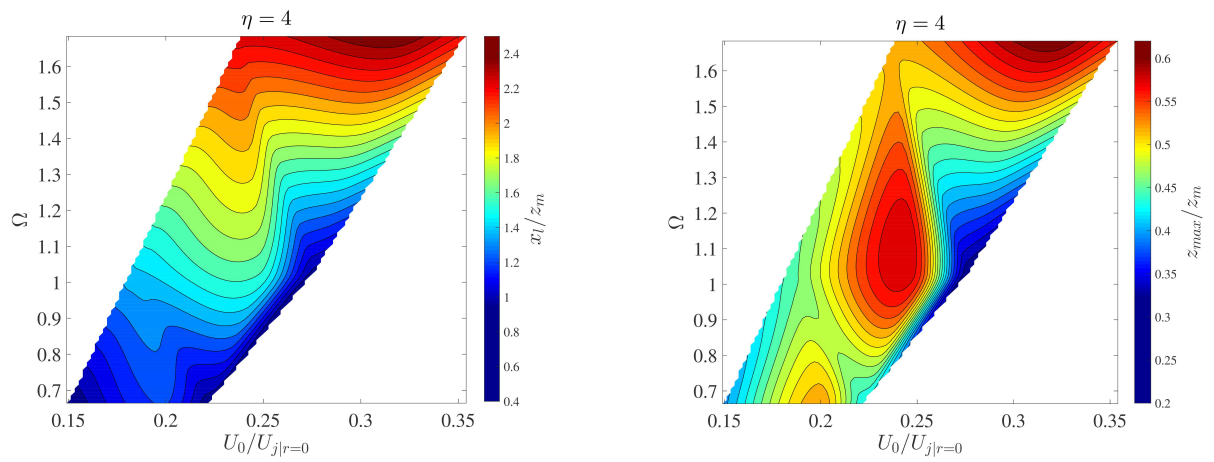

Figure 22: Contour plots of the mean $x_{l} / z_{m}$ (left) and the mean $z_{\max } / z_{m}$ (right) for model firebrands with aspect ratio $\eta=4$ within the parameter space of $\Omega-U_{0} / U_{j \mid r=0}$
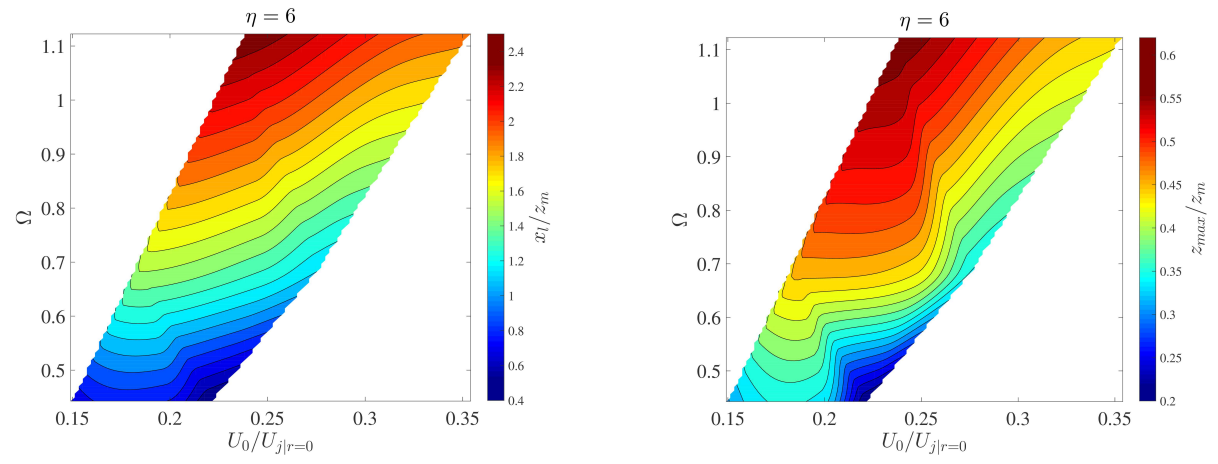

Figure 23: Contour plots of the mean $x_{l} / z_{m}$ (left) and the mean $z_{\max } / z_{m}$ (right) for model firebrands with aspect ratio $\eta=6$ within the parameter space of $\Omega-U_{0} / U_{j \mid r=0}$ 
[1] Albertson, M. L., Y. Dai, R. Jensen, and H. Rouse (1950). Diffusion of submerged jets. Transactions of the American Society of Civil Engineers 115(1), 639-664.

[2] Albini, F. A. (1962). Scattering of plane wave by inhomogeneous sphere under born approximation. Journal of Applied Physics 33(10), 3032-3036.

[3] Albini, F. A. (1964). Approximate evaluation of slender radome performance in hypersonic flight. AIAA Journal 2(3), 476-483.

[4] Albini, F. A. (1979). Spot fire distance from burning trees : a predictive model. United States.

[5] Albini, F. A. (1981). Spot fire distance from isolated sources : extensions of a predictive model. United States.

[6] Albini, F. A. (1983a). Transport of Firebrands by Line Thermals. Combustion Science and Technology 32(5-6), 277-288.

[7] Albini, F. A. (1993, mar). Dynamics and modeling of vegetation fire. Observations. In Proceedings of the Workshop on Fire in the Environment, Berlin, Germany, pp. 39. Publ by John Wiley \& Sons Ltd.

[8] Albini, F. A. and R. G. Jahn (1961). Reflection and transmission of electromagnetic waves at electron density gradients. Journal of Applied Physics 32(1), 75-82.

[9] Albini, F. A. F. A. . (1983b). Potential spotting distance from wind-driven surface fires / Frank A. Albini.

[10] Anthenien, R. A., S. D. Tse, and A. C. Fernandez-Pello (2006). On the trajectories of embers initially elevated or lofted by small scale ground fire plumes in high winds. Fire Safety Journal 41(5), 349-363.

[11] Barr, B. W. and O. Ezekoye (2013, jan). Thermo-mechanical modeling of firebrand breakage on a fractal tree. Proceedings of the Combustion Institute 34(2), 2649-2656. 
[12] Bell, W. G. (1971). The Great Fire of London in 1666. Greenwood Publishing Group.

[13] Bhutia, S., M. A. Jenkins, and R. Sun (2010, mar). Comparison of firebrand propagation prediction by a plume model and a coupledfire/atmosphere largeeddy simulator. Journal of Advances in Modeling Earth Systems 2, 4.

[14] Cermak, J. E. and N. Isyumov (1998). Wind tunnel studies of buildings and structures. American Society of Civil Engineers.

[15] Fischer, H. B., E. J. List, R. C. Y. Koh, J. Imberger, N. H. Brooks, and H. B. Fisher (1979). Mixing in inland and coastal waters. Academic.

[16] Gonzalez, R. C. (2009). Digital image processing. Pearson Education India.

[17] Gonzalez, R. C., R. E. Woods, and S. L. Eddins (2004). Digital image processing using MATLAB. Pearson Education India.

[18] Gould, J., W. McCaw, N. Cheney, P. Ellis, I. Knight, and A. Sullivan (2008). Project Vesta: fire in dry eucalypt forest: fuel structure, fuel dynamics and fire behaviour. CSIRO PUBLISHING.

[19] Grayson, M., W. Pang, and S. Schiff (2012, mar). Three-dimensional probabilistic wind-borne debris trajectory model for building envelope impact risk assessment. Journal of Wind Engineering and Industrial Aerodynamics 102, $22-35$.

[20] Himoto, K. and T. Tanaka (2005). Transport of disk-shaped firebrands in a turbulent boundary layer author. In the Eighth International Symposium on Fire Safety Science, pp. 18--23.

[21] Himoto, K. and T. Tanaka (2008). Development and validation of a physicsbased urban fire spread model. Fire Safety Journal 43(7), 477-494.

[22] Holmes, J., C. Baker, and Y. Tamura (2006). Tachikawa number: a proposal. Journal of Wind Engineering and Industrial Aerodynamics 94(1), 4147. 
[23] Karimpour, A. and N. B. Kaye (2012, January). On the stochastic nature of compact debris flight. J. Wind Eng. \& Ind. Aero. 100(1), 77-90.

[24] Koo, E., R. R. Linn, P. J. Pagni, and C. B. Edminster (2012). Modelling firebrand transport in wildfires using HIGRAD/FIRETEC. International Journal of Wildland Fire 21(4), 396-417.

[25] Koo, E., P. J. Pagni, D. R. Weise, and J. P. Woycheese (2010). Firebrands and spotting ignition in large-scale fires. International Journal of Wildland Fire $19(7), 818$.

[26] Lee, J. H. W. and V. H. Chu (2003). Turbulent jets and plumes: A Lagrangian approach. Springer.

[27] Lee, S.-L. and J. Hellman (1969). Study of firebrand trajectories in a turbulent swirling natural convection plume. Combustion and Flame 13(6), 645-655.

[28] Lee, S.-L. and J. M. Hellman (1970). Firebrand trajectory study using an empirical velocity-dependent burning law. Combustion and Flame 15(3), 265-274.

[29] Lin, N., J. D. Holmes, and C. W. Letchford (2007, feb). Trajectories of Wind-Borne Debris in Horizontal Winds and Applications to Impact Testing. J. Struct. Eng. 133(2), 274-282.

[30] Liu, Z., D. Prevatt, L. Aponte-Bermudez, K. Gurley, T. Reinhold, and R. Akins (2009). Field measurement and wind tunnel simulation of hurricane wind loads on a single family dwelling. Engineering Structures 31(10), 22652274 .

[31] Manzello, S. L., T. G. Cleary, J. R. Shields, A. Maranghides, W. Mell, and J. C. Yang (2008). Experimental investigation of firebrands: Generation and ignition of fuel beds. Fire Safety Journal 43(3), 226-233. 
[32] Manzello, S. L., A. Maranghides, and W. E. Mell (2007). Firebrand generation from burning vegetation. International Journal of Wildland Fire 16(4).

[33] Manzello, S. L., A. Maranghides, W. E. Mell, T. G. Cleary, and J. C. Yang (2006). Firebrand production from burning vegetation. Forest Ecology and Management 234, S119-S119.

[34] Manzello, S. L., A. Maranghides, J. R. Shields, W. E. Mell, Y. Hayashi, and D. Nii (2009). Mass and size distribution of firebrands generated from burning Korean pine (Pinus koraiensis) trees. Fire and Materials 33(1).

[35] Mell, W. E., S. L. Manzello, A. Maranghides, D. Butry, and R. G. Rehm (2010). The wildland-urban interface fire problem-current approaches and research needs. International Journal of Wildland Fire 19(2), 238-251.

[36] Nielsen, H. and L. Tao (1965). The fire plume above a large free-burning fire. In Symposium (International) on Combustion, Volume 10, pp. 965-972. Elsevier.

[37] Richards, P. J. (2010). Steady Aerodynamics of Rod and Plate Type Debris.pdf. In 17th Australian Fluid Mechanics Conference.

[38] Richards, P. J., N. Williams, B. Laing, M. McCarty, and M. Pond (2008, October). Numerical calculation of the three-dimensional motion of windborne debris. Journal of Wind Engineering and Industrial Aerodynamics 96(10), 2188-2202.

[39] Sardoy, N., J. L. Consalvi, a. Kaiss, a. C. Fernandez-Pello, B. Porterie, and a.C. Fernandez-Pello (2008, aug). Numerical study of ground-level distribution of firebrands generated by line fires. Combustion and Flame 154(3), $478-488$.

[40] Sardoy, N., J.-L. Consalvi, B. Porterie, and A. Fernandez-Pello (2007). Modeling transport and combustion of firebrands from burning trees. Combustion and Flame 150(3), 151-169. 
[41] Sardoy, N., J.-L. Consalvi, B. Porterie, and A. Kaiss (2006). Transport and combustion of Ponderosa Pine firebrands from isolated burning trees. In 2006 1st International Symposium on Environment Identities and Mediterranean Area, ISEIM, July 9, 2006 - July 12, Corte-Ajaccio, France, pp. 611. Polytech'Marseille, Dept. of Mechanical Engineering, CNRS UMR 6595, Technopole Chateau-Gombert, 5 rue Enrico Fermi, 13453 Marseille, France: Inst. of Elec. and Elec. Eng. Computer Society.

[42] Scaramuzza, D., A. Martinelli, and R. Siegwart (2006). A toolbox for easily calibrating omnidirectional cameras. In 2006 IEEE/RSJ International Conference on Intelligent Robots and Systems, pp. 5695-5701. IEEE.

[43] Song, J., X. Huang, N. Liu, H. Li, and L. Zhang (2016). The Wind Effect on the Transport and Burning of Firebrands. Fire Technology.

[44] Tarifa, C. S., P. P. Del Notario, F. G. Moreno, and A. R. Villa (1967). Transport and combustion of firebrands, final report of grants FG-SP-11 and FG-SP-146. US Department of Agriculture Forest Service.

[45] Tarifa, C. S., P. Notario, and F. G. Moreno (1965). On the flight paths and lifetimes of burning particles of wood. In Symposium (international) on combustion, Volume 10, pp. 1021-1037. Elsevier.

[46] Tavoularis, S. (2005). Measurement in fluid mechanics. Cambridge University Press.

[47] Tohidi, A. (2016). Experimental and Numerical Modeling of Wildfire Spread via Fire Spotting. Doctoral dissertation, Clemson University.

[48] Tohidi, A. and N. Kaye (2016a, November). Experimental \& numerical modeling of non-combusting model firebrands' transport. In Bulletin of the $69^{\text {th }}$ American Physical Society, Division of Fluid Dynamics, Volume 61. American Physical Society (APS).

[49] Tohidi, A., N. Kaye, and W. Bridges (2015). Statistical description of firebrand size and shape distribution from coniferous trees for use in Metropolis 
643

Monte Carlo simulations of firebrand flight distance. Fire Safety Journal 77, 21-35.

[50] Tohidi, A. and N. B. Kaye (2013). The Sensitivity of Modeled Flight Distance to the Lofting to Transport Transition Criterion in Coupled Ember Flight Models. In 12th Americas Conference on Wind Engineering, Seattle, WA, USA, pp. 433-448.

[51] Tohidi, A. and N. B. Kaye (2016b). Highly buoyant bent-over plumes in a boundary layer. Atmospheric Environment 131, 97-114.

[52] Tse, S. D. and A. C. Fernandez-Pello (1998). On the flight paths of metal particles and embers generated by power lines in high windsa potential source of wildland fires. Fire Safety Journal 30(4), 333-356. 